\title{
Noise in gravitational-wave detectors and other classical-force measurements is not influenced by test-mass quantization
}

\author{
Vladimir B. Braginsky, ${ }^{1}$ Mikhail L. Gorodetsky, ${ }^{1}$ Farid Ya. Khalili, ${ }^{1}$ Andrey B. Matsko, ${ }^{2}$ Kip S. Thorne, ${ }^{3}$ \\ and Sergey P. Vyatchanin ${ }^{1}$ \\ ${ }^{1}$ Physics Faculty, Moscow State University, Moscow, Russia \\ ${ }^{2}$ Department of Physics, Texas A\&M University, College Station, Texas 77843-4242 \\ ${ }^{3}$ Theoretical Astrophysics, California Institute of Technology, Pasadena, California 91125
}

(Received 2 September 2001; published 7 April 2003)

\begin{abstract}
It is shown that photon shot noise and radiation-pressure back-action noise are the sole forms of quantum noise in interferometric gravitational wave detectors that operate near or below the standard quantum limit, if one filters the interferometer output appropriately. No additional noise arises from the test masses' initial quantum state or from reduction of the test-mass state due to measurement of the interferometer output or from the uncertainty principle associated with the test-mass state. Two features of interferometers are central to these conclusions: (i) The interferometer output [the photon number flux $\hat{\mathcal{N}}(t)$ entering the final photodetector] commutes with itself at different times in the Heisenberg picture, $\left[\hat{\mathcal{N}}(t), \hat{\mathcal{N}}\left(t^{\prime}\right)\right]=0$ and thus can be regarded as classical. (ii) This number flux is linear to high accuracy in the test-mass initial position and momentum operators $\hat{x}_{o}$ and $\hat{p}_{o}$, and those operators influence the measured photon flux $\hat{\mathcal{N}}(t)$ in manners that can easily be removed by filtering. For example, in most interferometers $\hat{x}_{o}$ and $\hat{p}_{o}$ appear in $\hat{\mathcal{N}}(t)$ only at the test masses' $\sim 1 \mathrm{~Hz}$ pendular swinging frequency and their influence is removed when the output data are high-pass filtered to get rid of noise below $\sim 10 \mathrm{~Hz}$. The test-mass operators $\hat{x}_{o}$ and $\hat{p}_{o}$ contained in the unfiltered output $\hat{\mathcal{N}}(t)$ make a nonzero contribution to the commutator $\left[\hat{\mathcal{N}}(t), \hat{\mathcal{N}}\left(t^{\prime}\right)\right]$. That contribution is precisely canceled by a nonzero commutation of the photon shot noise and radiation-pressure noise, which also are contained in $\hat{\mathcal{N}}(t)$. This cancellation of commutators is responsible for the fact that it is possible to derive an interferometer's standard quantum limit from test-mass considerations, and independently from photon-noise considerations, and get identically the same result. These conclusions are all true for a far wider class of measurements than just gravitational-wave interferometers. To elucidate them, this paper presents a series of idealized thought experiments that are free from the complexities of real measuring systems.
\end{abstract}

DOI: 10.1103/PhysRevD.67.082001 PACS number(s): 04.80.Nn, 03.65.Ta, 42.50.Lc, 95.55.Ym

\section{QUESTIONS TO BE ANALYZED AND SUMMARY OF ANSWERS}

It has long been known that the Heisenberg uncertainty principle imposes a "standard quantum limit" (SQL) on high-precision measurements [1-3]. This SQL can be circumvented by using "quantum nondemolition" (QND) techniques [2-9].

For broad-band interferometric gravitational-wave detectors the SQL is a limiting (single-sided) spectral density

$$
S_{h}(f)=\frac{8 \hbar}{m(2 \pi f)^{2} L^{2}}
$$

for the gravitational-wave field $h(t)[10,11]$. Here $\hbar$ is Planck's constant divided by $2 \pi, m$ is the mass of each of the interferometer's four test masses, $L$ is the interferometer's arm length, and $f$ is frequency.

This SQL firmly constrains the sensitivity of all conventional interferometers [interferometers with the same optical topology as the Laser Interferometric Gravitational Wave Observatory's (LIGO's) first-generation gravitational-wave detectors] $[12,13]$. LIGO's second-generation interferometers (LIGO-II; ca. 2008) are expected to reach this SQL for their $m=40 \mathrm{~kg}$ test masses in the vicinity of $f \sim 100 \mathrm{~Hz}$ [14], and may even beat it by a modest amount thanks to a "signal recycling mirror" that converts them from conventional interferometers into QND devices [15-17]. LIGO-III interferometers are likely to beat the SQL by a factor $\sim 4$ or more; see, e.g., [13].

In the research and development for LIGO-II interferometers [14-17] and in the attempts to invent strongly QND LIGO-III interferometers [18-24,13], it is important to understand clearly the physical nature of the quantum noise which imposes the SQL, and to be able to compute with confidence the spectral density of this quantum noise for various interferometer designs. These issues are the subject of this paper.

There are two standard ways to derive the gravitationalwave SQL (1.1), and correspondingly two different viewpoints on it. The first derivation $[10,25]$ focuses on the quantum mechanics of the interferometer's test masses and ignores the interferometer's other details. In the simplest version of this derivation, one imagines a sequence of instantaneous measurements of the difference

$$
\hat{x} \equiv\left(\hat{x}_{1}-\hat{x}_{2}\right)-\left(\hat{x}_{3}-\hat{x}_{4}\right)
$$

of the center-of-mass positions of the four test masses, and from this measurement sequence one infers the changes of $x$ and thence the time varying gravitational-wave field $h(t)$ $=x(t) / L$. At time $t$ immediately after one of the measure- 
ments, the test masses' reduced state has position variance $[\Delta x(t)]^{2}$ no smaller than the measurement's accuracy. During the time interval $\tau=t^{\prime}-t$ between this measurement and the next, the test masses are free, so $\hat{x}(t)$ evolves as the position of a free particle with mass

$$
\mu=m / 4
$$

[the reduced mass of the four-body system with relative position (1.2)]. The Heisenberg-Picture commutation relations for a free particle

$$
\left[\hat{x}(t), \hat{x}\left(t^{\prime}\right)\right]=\frac{i \hbar\left(t^{\prime}-t\right)}{\mu}=\frac{4 i \hbar \tau}{m}
$$

imply that, whatever may be the state of the test masses, the variance $\left[\Delta x\left(t^{\prime}\right)\right]^{2}$ of $\hat{x}$ just before the next measurement must satisfy the Heisenberg uncertainty relation

$$
\Delta x(t) \Delta x\left(t^{\prime}\right) \geqslant \frac{\hbar\left|t-t^{\prime}\right|}{2 \mu}=\frac{2 \hbar \tau}{m} .
$$

The accuracy with which the change of $x$ between $t$ and $t^{\prime}$ can be measured is no better than the value obtained by setting $\Delta x(t)=\Delta x\left(t^{\prime}\right)$, and in classical language that accuracy is related to the minimum possible spectral density of the noise at frequency $f \simeq 1 / \pi \tau$ by $\Delta x(t)=\Delta x\left(t^{\prime}\right)$ $\simeq \sqrt{S_{h}(f) / \tau}$. Simple algebra then gives expression (1.1) for the SQL of $S_{h}(f)$. A more sophisticated analysis [10], based on measurements that are continuous rather than discrete and on a nonunitary Feynman-path-integral evolution of the testmass state [26,27], gives precisely the SQL (1.1).

The second derivation of the SQL $[28,29]$ ignores the quantum mechanics of the test mass, and focuses instead on that of the laser light which monitors the test-mass motion. The light produces two kinds of noise: photon shot noise, which gets superposed on the output gravitational-wave signal, and radiation-pressure fluctuations, which produce a random back-action force on the test masses, thereby influencing their position evolution and thence the interferometer output. In an ideal, SQL-limited interferometer, both noises-shot and radiation-pressure-arise from quantum electrodynamic vacuum fluctuations that enter the interferometer through its dark port and superpose on the highly classical laser light $[28,29]$. The radiation-pressure spectral density is proportional to the laser-light power $P$, the shotnoise spectral density is proportional to $1 / P$, and their product is independent of $P$ and is constrained by the uncertainty principle for light (or equivalently by the electromagnetic field commutation relations) to be no smaller than

$$
S_{x} S_{F}=\hbar^{2}
$$

[cf. Eqs. (6.7) and (6.17) of [3] in which there is a factor $1 / 4$ on the right side because Ref. [3] uses a double-sided spectral density, while the present paper uses the gravity-wave community's single-sided convention]. In Eq. (1.6) $S_{x}(f)$ is the spectral density of the shot noise that is superposed on the interferometer's output position signal $x(t), S_{F}(f)$ is the spectral density of the radiation-pressure force that acts on the test-mass center-of-mass degree of freedom $x$, and we have assumed that the shot noise and radiation-pressure force are uncorrelated as is the case for conventional (LIGO-I type) interferometers $[13,15-17]$. At frequency $f$ the test mass responds to the Fourier component $\widetilde{F}(f)$ of the force with a position change $\tilde{x}(f)=-\widetilde{F}(f) /\left[\mu(2 \pi f)^{2}\right]$, and correspondingly the net gravitational-wave noise is

$$
S_{h}(f)=\frac{1}{L^{2}}\left(S_{x}+\frac{S_{F}}{\mu^{2}(2 \pi f)^{4}}\right) .
$$

By combining Eqs. (1.6), (1.7) and (1.3), we obtain the SQL (1.1) for a conventional interferometer, e.g. LIGO-I.

In view of these two very different derivations of the SQL, test-mass quantization and light quantization, three questions arise: (i) Are the test-mass quantization and the light quantization just two different viewpoints on the same physics? - in which case the correct SQL is Eq. (1.1). Or are they fully or partially independent effects? - in which case we would expect their noises to add, causing the true SQL for $S_{h}$ to be larger by, perhaps, a factor 2 [and thence the event rate in an SQL-limited interferometer to be reduced by a factor $\sim(\sqrt{2})^{3} \simeq 3$ ]. (ii) How should one compute the quantum noise in candidate designs for the QND LIGO-II and LIGO-III interferometers? One inevitably must pay close attention to the behavior of the light (and thus also its quantization), since the optical configuration will differ markedly from one candidate design to another. Must one also pay close attention to the quantum mechanics of the test masses, including their commutation relation (1.4) and the continual reduction of their state as information about them is continually put onto the light's modulations and then measured? (iii) Similarly, how should one design a QND interferometer? Need one adjust one's design so as to drive both the light's noise and the test-mass noise below the SQL?

As we shall show, the answers are these: (ii) The testmass quantization is irrelevant to the interferometer's noise and correspondingly test-mass state reduction is irrelevant, if one filters the output data appropriately. (For interferometers with conventional optical topology such as LIGO-I, it is sufficient to discard all data near the test masses' $\sim 1 \mathrm{~Hz}$ swinging frequency.) Therefore, one can ignore test-mass quantization and state reduction when computing the noise of a candidate interferometer. (iii) Similarly, one can ignore the test mass's quantum noise when designing a QND interferometer that beats the SQL. One need only pay attention to the light's quantum noise, and in principle, by manipulating the light appropriately (and filtering the output data appropriately), one can circumvent the SQL completely. (i) Correspondingly, the SQL (1.1) as derived from light quantization is precisely correct; there is no extra factor 2 caused by testmass quantization. [The fact that one can also derive the SQL from test-mass quantization is a result of an intimate connection between the uncertainty principles for a measured system (the test masses in our case) and the system that makes the measurement (the light). We shall elucidate this intimate connection from one viewpoint at the end of Sec. II B 4 . From another viewpoint, it is due to the fact that the com- 
mutator $\left[\hat{x}(t), \hat{x}\left(t^{\prime}\right)\right]$, which underlies the test-mass derivation (1.4), (1.5) of the SQL, also underlies the derivation of the measuring light's uncertainty relation (1.6); see the role of the generalized susceptibility $\chi\left(t, t^{\prime}\right)=(1 / i \hbar)\left[\hat{x}\left(t^{\prime}\right)\right.$, $\hat{x}(t)]$ in Sec. 6.3 of Ref. [3].]

Central to our answers (i), (ii) and (iii) is the fact that an interferometric gravitational-wave detector does not monitor the time-evolving test-mass position $\hat{x}(t)$. Rather, it only monitors classical changes in $\hat{x}(t)$ induced by the classical gravitational-wave field $h(t)$ and other classical ${ }^{1}$ forces (thermal, seismic, ...) acting on the test masses, and it does so without extracting information about the actual quantized position $\hat{x}(t)$. The detector has a classical input $[h(t)]$ and a classical output $[h(t)$ contaminated by noise that (as we shall see) commutes with itself at different times and that therefore can be regarded as a time-evolving $c$ number]. The quantum properties of the test masses and the light are merely intermediaries through which the classical signal must pass. This would not be the case for a device designed to make a sequence of absolute measurements of the quantum mechanical position $\hat{x}(t)$.

Our answers (i), (ii), (iii) hold true for a far wider range of measuring devices than just interferometric gravitationalwave detectors. They hold quite generally for any welldesigned device that measures a classical force acting on any quantum mechanical system. In particular, they remain true if the device makes measurements that are linear in the sense of Appendix B, and one filters the device's output to remove all information at the natural frequencies of the quantum system's dynamics (e.g. at its eigenfrequency if the quantum system is a harmonic oscillator).

(While this paper was under consideration for publication, we became aware of a beautiful path-integral analysis by Caves (Sec. III C of [26]) which elucidates answers like our (i), (ii), (iii) for a wide class of measurements of a harmonic oscillator, on which a classical force is acting. Caves' Ref. [26] contains important insights. We strongly recommend it to all readers of our paper.)

In Sec. II we will elucidate our answers (i), (ii), (iii) by considering pedagogical examples of idealized devices that make discrete, quick measurements on a test mass. These examples will reveal two central underpinnings of our answers: (a) the vanishing of the measurement's "output commutators"-i.e., the commutators of the observables (Hermitian operators) that represent the entries in the output data stream, and (b) a data-processing procedure that removes from the data all influence of the test-mass quantum observables (initial position $\hat{x}_{o}$ and initial momentum $\hat{p}_{o}$ ). Our examples will also elucidate two strategies for beating the SQL: (A) put the measuring apparatus ("meters") into specially chosen initial states (the analog of squeezed states), and (B) measure a wisely chosen linear combination of po-

\footnotetext{
${ }^{1}$ All these forces-gravitational-wave, thermal, seismic, etc.actually do have a quantum component, but in practice their levels of excitation are so large that we can regard them as classical.
}

sition and momentum for the test mass and thereby remove the effects of the meters' back action from the output data (make a "quantum variational measurement").

Our examples are the following: We will begin in Sec. II A with a simple, idealized, instantaneous single measurement of the position of a single test mass. This example will demonstrate that the noise associated with test-mass quantization and the noise associated with the meter's quantization are truly independent (though closely linked), and will illustrate how under some circumstances they can add, producing a doubling of the noise power. Then, in Sec. II B, we will analyze the use of a sequence of these idealized, instantaneous position measurements to monitor a classical force that acts on the test mass. This example will illustrate the vanishing self-commutator of the output data samples, which arises from a cancellation of the test-mass-position commutator by the measurement-noise commutator; it also will illustrate how signal processing can remove all influence of test-mass quantization and test-mass state reduction from the output data stream. Our third example (Sec. II C) will be a Heisenberg-microscope-like realization of these instantaneous, idealized position measurements, in which a pulse of near-monochromatic light is reflected off the test mass, thereby encoding the test-mass position in a phase shift of the light. This example will give reality to the idealized examples in Secs. II A and II B, and will help connect them to the subsequent discussion of interferometric gravitationalwave detectors.

In Sec. III we will use the insights from our pedagogical examples to prove and elucidate our three answers [(i), (ii), (iii) above] for gravitational-wave interferometers, and also for a wide range of other classical force measurements. The underpinnings for our answers will be: (a) a proof that for a quantized electromagnetic wave, such as that entering the final photodetector of an interferometer, the photon number flux operator commutes with itself at different times (this flux is the output data stream), and (b) a proof that all influence of the test-mass quantum observables can be removed from the output data stream by appropriate filtering, and for conventional interferometers it is sufficient to remove all data near the test masses' $\sim 1 \mathrm{~Hz}$ swinging frequency, e.g. by the kind of high-pass filtering that is routinely used in gravitational-wave detectors. Our analysis will also elucidate QND interferometer designs based on (A) squeezed-input states for light and (B) variational-output measurements.

The issues studied in this paper are most efficiently analyzed in the Heisenberg picture, and the Heisenberg picture gives particularly clear insights into them. For this reason, we will use the Heisenberg picture throughout the body of this paper. Readers who are uncomfortable with the Heisenberg picture may find Appendix A reassuring; there we will give a detailed Schrödinger-picture analysis of the most important of our pedagogical examples, that of Sec. II B.

\section{PEDAGOGICAL EXAMPLES}

\section{A. A single position measurement: "Double" uncertainty relation}

We begin with a simple pedagogical example of a single measurement of the position of a single test mass. The Heisenberg microscope is a famous realization of this example; see Sec. II C. 
The measurement is idealized as instantaneous and as occurring at time $t=0$. At times arbitrarily close to $t=0$, the Hamiltonian for the test mass (with position and momentum $\hat{x}$ and $\hat{p}$ ) and the measuring device (the meter, with generalized position $\hat{Q}$ and generalized momentum $\hat{P}$ ) is

$$
H=\frac{\hat{p}^{2}}{2 \mu}-\delta(t) \hat{x} \hat{P}+\frac{\hat{P}^{2}}{2 M} .
$$

Here $\delta(t)$ is the Dirac delta function, $\mu$ is the test mass's mass and $M$ is the generalized mass of the meter. For pedagogical simplicity we make $M$ arbitrarily large so $\hat{Q}$ and $\hat{P}$ do not evolve in the Heisenberg picture except at the moment of interaction, and correspondingly we rewrite the Hamiltonian as

$$
H=\frac{\hat{p}^{2}}{2 \mu}-\delta(t) \hat{x} \hat{P} .
$$

A simple calculation in the Heisenberg picture gives the following expressions for the positions and momenta immediately after the measurement, in terms of those immediately before:

$$
\begin{aligned}
& \hat{P}_{\text {after }}=\hat{P}_{\text {before }}, \\
& \hat{x}_{\text {after }}=\hat{x}_{\text {before }}, \\
& \hat{Q}_{\text {after }}=\hat{Q}_{\text {before }}-\hat{x}_{\text {before }} \\
& \hat{p}_{\text {after }}=\hat{p}_{\text {before }}+\hat{P}_{\text {before }} .
\end{aligned}
$$

The meter's generalized position $\hat{Q}_{\text {after }}$ is amplified and read out classically immediately after the interaction, to determine the test-mass position. The resulting measured position, expressed as an operator, is $\hat{x}_{\text {meas }} \equiv-\hat{Q}_{\text {after }}=\hat{x}_{\text {before }}-\hat{Q}_{\text {before }}$ [Eq. (2.3c)], and the measurement leaves the actual test-mass position operator unperturbed [Eq. (2.3b)] but it perturbs the test-mass momentum [Eq. (2.3d)].

It is instructive to rewrite Eqs. (2.3c) and (2.3d) in the form

$$
\begin{aligned}
& \hat{x}_{\text {meas }}=\hat{x}_{\text {before }}+\delta \hat{x}_{\text {meas }}, \\
& \hat{p}_{\text {after }}=\hat{p}_{\text {before }}+\delta \hat{p}_{\text {BA }},
\end{aligned}
$$

with

$$
\delta \hat{x}_{\text {meas }}=-\hat{Q}_{\text {before }}, \quad \delta \hat{p}_{\mathrm{BA}}=+\hat{P}_{\text {before }} .
$$

The simple equations (2.4a), (2.4b) embody the measurement result and its back action; $\hat{x}_{\text {meas }}$ is the measured value of $\hat{x}_{\text {before }}=\hat{x}_{\text {after }}, \delta \hat{x}_{\text {meas }}$ is the noise superposed on that measured value by the meter, and $\delta \hat{p}_{\mathrm{BA}}$ is the back-action impulse given to the test mass by the meter. Equations (2.4) are actually much more general than our simple example; they apply to any sufficiently quick, "linear" measurement; see Eqs. (5.2), (5.14) and (5.23) of Ref. [3], and see Appendix B below.

The initial test-mass position and momentum and the initial meter position and momentum have the usual commutation relations,

$$
\left[\hat{x}_{\text {before }}, \hat{p}_{\text {before }}\right]=i \hbar=\left[\hat{Q}_{\text {before }}, \hat{P}_{\text {before }}\right] \text {. }
$$

The second of these and Eqs. (2.5) imply that the measurement noise $\delta \hat{x}_{\text {meas }}$ and the back-action impulse $\delta \hat{p}_{\mathrm{BA}}$ have this same standard commutator, but with the sign reversed

$$
\left[\delta \hat{x}_{\text {meas }}, \delta \hat{p}_{\mathrm{BA}}\right]=-i \hbar
$$

This has an important implication: The measured value of the test-mass position and the final value of the test-mass momentum commute:

$$
\left[\hat{x}_{\text {meas }}, \hat{p}_{\text {after }}\right]=0
$$

This result, like the simple measurement and back-action equations $(2.4 \mathrm{a}),(2.4 \mathrm{~b})$, is true not only for this pedagogical example, but also for any other sufficiently quick, linear measurement; see, e.g., Sec. II C below.

It is evident from Eqs. (2.4) and (2.5) that the variances of $\hat{x}_{\text {meas }}$ and $\hat{p}_{\text {after }}$ are influenced by the initial states of both the meter and the test mass:

$$
\begin{aligned}
& \left(\Delta x_{\text {meas }}\right)^{2}=\left(\Delta x_{\text {before }}\right)^{2}+\left(\Delta Q_{\text {before }}\right)^{2}, \\
& \left(\Delta p_{\text {after }}\right)^{2}=\left(\Delta p_{\text {before }}\right)^{2}+\left(\Delta P_{\text {before }}\right)^{2} .
\end{aligned}
$$

Here we have assumed, as is easy to arrange, that the initial states of the meter and the test mass are uncorrelated. Now, the initial states of the test mass and meter are constrained by the uncertainty relations

$$
\begin{gathered}
\Delta x_{\text {before }} \cdot \Delta p_{\text {before }} \geqslant \frac{\hbar}{2}, \\
\Delta Q_{\text {before }} \cdot \Delta P_{\text {before }} \geqslant \frac{\hbar}{2},
\end{gathered}
$$

which follow from the commutators (2.6). From the viewpoint of the measurement equations (2.4a), (2.4b), the meter equation (2.12) is an uncertainty relation between the noise $\delta \hat{x}_{\text {meas }}=-\hat{Q}_{\text {before }}$ that the meter superimposes on the output signal, and the back-action impulse $\delta \hat{p}_{\mathrm{BA}}=\hat{P}_{\text {before }}$ that the meter gives to the test mass. In the Heisenberg microscope, $\delta \hat{x}_{\text {meas }}$ would be photon shot noise and $\delta \hat{p}_{\text {BA }}$ would be radiation-pressure impulse.

\footnotetext{
${ }^{2}$ I.e., quick compared to the evolution of the wave function of the measured quantity, so it can be regarded as constant during the measurement.
} 
The test-mass uncertainty relation (2.11) and meter uncertainty relation (2.12) both constrain the product of the measurement error (2.9) and the final momentum uncertainty (2.10), and by equal amounts. The result is a "doubling" of the uncertainty relation, so

$$
\Delta x_{\text {meas }} \cdot \Delta p_{\text {after }} \geqslant 2 \cdot \frac{\hbar}{2} \text {. }
$$

This doubling of the uncertainty relation relies crucially on our assumption that the initial states of the test mass and meter are uncorrelated. Correlations can produce a violation of the uncertainty relation (2.13). For example, initial correlations can be arranged so as to produce (in principle) a vanishing total measurement error $\Delta x_{\text {meas }}=0$ and a finite $\Delta p_{\text {after }}$ so the product $\Delta x_{\text {meas }} \cdot \Delta p_{\text {after }}$ vanishes-a result permitted by the vanishing commutator (2.8).

\section{B. Monitoring a classical force: "Single" uncertainty relation}

As we emphasized in Sec. I, the goal of LIGO-type detectors is not to measure any observables of a test mass, but rather to monitor an external force that acts on it. Correspondingly, it is desirable to design the measurement so the output is devoid of any information about the test mass's initial state. As we shall see, this is readily done in a way that removes the initial-state information during data processing. The result is a "single" uncertainty relation: the measurement result is influenced only by the quantum properties of the meter and not by those of the test mass.

\section{Von Neumann's thought experiment}

We illustrate this by a variant of a thought experiment devised by von Neumann [30] and often used to illustrate issues in the quantum theory of measurement; see, e.g., [31] and references therein. We analyze this thought experiment using the Heisenberg picture in the body of this paper, and we give a Schrödinger-picture analysis in Appendix A.

Our von Neumann thought experiment is a simple generalization of the position measurement described above. Specifically, we consider a free test mass, with mass $\mu$, position $\hat{x}$ and momentum $\hat{p}$, on which acts a classical force $F(t)$. To monitor $F(t)$, we probe the test mass instantaneously at times $t=0, \tau, \ldots,(N-1) \tau$ using $N$ independent meters labeled $r=0,1, \ldots, N-1$. Each meter is prepared in a carefully chosen state, it then interacts with the test mass, and then is measured. We filter the measurement results to deduce $F(t)$. Meter $r$ has generalized coordinate and momentum $\hat{Q}_{r}$ and $\hat{P}_{r}$, and its free Hamiltonian is vanishingly small, so $\hat{Q}_{r}$ and $\hat{P}_{r}$ do not evolve except at the moment of interaction. The total Hamiltonian for test mass plus classical force plus meters is

$$
\hat{H}=\frac{\hat{p}^{2}}{2 \mu}-F(t) \hat{x}-\sum_{r=0}^{N-1} \delta(t-r \tau) \hat{x} \hat{P}_{r} .
$$

We denote by $\hat{x}_{0}$ and $\hat{p}_{0}$ the test-mass position and momentum at time $t=0$ when the experiment begins, and by $\hat{x}_{r}$ and $\hat{p}_{r}$ their values immediately after interacting with meter $r$, at time $t=r \tau$. The momentum of meter $r$ is a constant of the motion, so we denote it by $\hat{P}_{r}$ at all times. The meter coordinate changes due to the interaction; we denote its value before the interaction by $\hat{Q}_{r}^{\text {before }}$ and after the interaction by $\hat{Q}_{r}$.

It is easy to show, from the Heisenberg equations for the Hamiltonian (2.14), that the test-mass position immediately after its $r$ 'th interaction is

$$
\hat{x}_{r}=\hat{x}_{o}+\frac{\hat{p}_{o}}{\mu} r \tau+\sum_{s=0}^{r} \hat{P}_{s} \frac{(r-s) \tau}{\mu}+\xi_{r} .
$$

Here the first two terms are the free evolution of the test mass, the third (with the sum) is the influence of the meters' back-action forces (analog of radiation-pressure force in an interferometer), and the fourth,

$$
\xi_{r} \equiv \frac{1}{\mu} \int_{0}^{r \tau} \int_{0}^{t} F\left(t^{\prime}\right) d t^{\prime} d t=\frac{1}{\mu} \int_{0}^{r \tau}\left(r \tau-t^{\prime}\right) F\left(t^{\prime}\right) d t^{\prime},
$$

is the effect of the classical force. The force $F(t)$ is encoded in the sequence of classical displacements $\left\{\xi_{1}, \xi_{2}, \ldots, \xi_{N}\right\}$. It is also easy to show from the Heisenberg equations that the meter's generalized coordinate after interaction with the test mass is

$$
\begin{aligned}
\hat{Q}_{r} & =\hat{Q}_{r}^{\text {before }}-\hat{x}_{r} \\
& =\hat{Q}_{r}^{\text {before }}-\hat{x}_{o}-\frac{\hat{p}_{o}}{\mu} r \tau-\sum_{s=0}^{r} \hat{P}_{s} \frac{(r-s) \tau}{\mu}-\xi_{r} .
\end{aligned}
$$

\section{Vanishing of the output's self-commutator}

The set of final meter coordinates $\vec{Q}$ $\equiv\left\{\hat{Q}_{0}, \hat{Q}_{1}, \ldots, \hat{Q}_{N-1}\right\}$ forms the final data string for data analysis. It has vanishing self-commutator,

$$
\left[\hat{Q}_{s}, \hat{Q}_{r}\right]=0 \text { for all } s \text { and } r
$$

- a result that can be deduced from the vanishing singlemeasurement commutator $\left[\hat{x}_{\text {meas }}, \hat{p}_{\text {after }}\right]=0$ [Eq. (2.8)] for the earlier of the two measurements.

It is instructive to see explicitly how this vanishing commutator arises, without explicit reference to our singlemeasurement analysis. The test-mass contributions to the $Q$ 's $\left[\hat{x}_{o}\right.$ and $\hat{p}_{o}$ in Eq. $\left.(2.17)\right]$ produce

$$
\begin{aligned}
{\left[\hat{Q}_{s}, \hat{Q}_{r}\right]_{\text {test mass }} } & =\left[-\hat{x}_{o}-\frac{\hat{p}_{o}}{\mu} s \tau,-\hat{x}_{o}-\frac{\hat{p}_{o}}{\mu} r \tau\right] \\
& =\frac{i \hbar(r-s) \tau}{\mu},
\end{aligned}
$$

which is the analog of Eq. (1.4) for an interferometer test mass. This must be cancelled by a contribution from the meters. Indeed it is. If (for concreteness) $r>s$, then the cancelling contribution comes from a commutator of (i) the 
$\hat{Q}_{s}^{\text {before }}$ piece of $\hat{Q}_{s}$ (the noise superposed on the output signal $s$ by meter $s$ ) and (ii) the $\hat{P}_{s}$ term in $\hat{Q}_{r}$ (the noise in the later measurement produced by the back-action of the earlier measurement):

$$
\begin{aligned}
{\left[\hat{Q}_{s}, \hat{Q}_{r}\right]_{\text {meter }} } & =\left[\hat{Q}_{s}^{\text {before }},-\hat{P}_{s} \frac{(r-s) \tau}{\mu}\right] \\
& =\frac{-i \hbar(r-s) \tau}{\mu}
\end{aligned}
$$

In this example, one can trace these cancellations to the bilinear form $\hat{x} \hat{P}_{s}$ and $\hat{x} \hat{P}_{r}$ of each piece of the interaction Hamiltonian. However, this type of cancellation is far more general than just bilinear Hamiltonians: In every sequence of measurements on any kind of system, by the time a human looks at the output data stream, its entries have all been amplified to classical size, and therefore they must all be classical quantities and must commute, $\left[\hat{Q}_{s}, \hat{Q}_{r}\right]=\left[Q_{s}, Q_{r}\right]$ $=0$. Remarkably, quantum mechanics is so constructed that, for a wide variety of measurements, the measured values (regarded as Hermitian observables) commute even before the amplification to classical size. This is true in the above example. It is true in a realistic variant of this example involving pulsed-light measurements (Sec. II C). It is true in a variant of this example involving continuous measurements by an electromagnetic wave in an idealized transmission line [32]. And, as we shall see in Sec. III A and Appendix C, it is also true for gravitational-wave interferometers-and indeed for all measurements in which the measured results are encoded in the photon number flux of a (quantized) electromagnetic wave; i.e., all measurements based on photodetection. More generally, it is true for any linear measurement [Appendix B below, Ref. [3], and Eq. (2.34) of Ref. [17]]; and, in fact, all the measurements discussed above, including gravitational-wave measurements, are linear.

The classical nature of the output signal (the commutation of the data entries) guarantees that, when a human looks at one data entry, the resulting reduction of the state of the measured system cannot have any influence on the observed values of the other data entries. Correspondingly, we can carry out any data processing procedures we wish on the $\hat{Q}_{r}$, without fear of introducing new quantum noise.

\section{Removal of test-mass influence from the output}

Our goal is to measure the classical force $F(t)$ that acted on the test mass, without any contamination from the test mass's quantum properties-more specifically, without any contamination from uncertainty-principle aspects of the test mass's initial state. The initial state does influence the measured values $\widetilde{Q}_{r}$ of the output observables $\hat{Q}_{r}$, since in the Heisenberg picture the $\hat{Q}_{r}$ contain the test mass's initial position $\hat{x}_{o}$ and momentum $\hat{p}_{o}$ [Eq. (2.17)]. Therefore, our goal translates into finding a data analysis procedure that will remove from the output data set $\left\{\widetilde{Q}_{1}, \widetilde{Q}_{2}, \ldots\right\}$ all influence of the test-mass initial state (or equivalently all influence of $\hat{x}_{o}$ and $\hat{p}_{o}$ ), while retaining the influence of $F(t)$. In fact, we can do so rather easily, regardless of what the test-mass initial state might have been. As we shall see, our ability to do so relies crucially on the linearity of our measurements; in particular, on the fact that the output observables $\hat{Q}_{r}$ are linear in $\hat{x}_{o}$ and $\hat{p}_{o}$.

To bring out the essence, we shall restrict ourselves to just three meters, $N=3$. The generalization to large $N$ is straightforward.

The measured data sample $\hat{Q}_{r}$ is equal to the freely evolving test-mass position at time $r \tau, \quad \hat{x}_{\text {free }}(t=r \tau)=\hat{x}_{o}$ $+\left(\hat{p}_{o} / \mu\right) r \tau$ (which is linear in $\hat{x}_{o}, \hat{p}_{o}$ ), plus noise. Since the free evolution satisfies the equation of motion $d^{2} \hat{x}_{\text {free }} / d t^{2}$ $=0$, it is a reasonable guess that we can remove the influence of $\hat{x}_{o}$ and $\hat{p}_{o}$ from the data $\widetilde{Q}_{r}$ by applying to them the discrete version of a second time derivative ${ }^{3}$ (which is a linear signal processing procedure). Accordingly, from the measured values $\left\{\widetilde{Q}_{0}, \widetilde{Q}_{1}, \widetilde{Q}_{2}\right\}$ of $\left\{\hat{Q}_{0}, \hat{Q}_{1}, \hat{Q}_{2}\right\}$ in a representative experiment, we construct the discrete second time derivative

$$
\widetilde{R}=\left(\widetilde{Q}_{2}-\widetilde{Q}_{1}\right)-\left(\widetilde{Q}_{1}-\widetilde{Q}_{0}\right)=\widetilde{Q}_{0}-2 \widetilde{Q}_{1}+\widetilde{Q}_{2}
$$

The following argument shows that all the statistical properties of this quantity, in a large series of experiments (in which the initial states $\mid$ in $\rangle$ of the test mass and meters are always the same) are, indeed, devoid of any influence of $\hat{x}_{o}$ and $\hat{p}_{o}$, and thus are unaffected by the test-mass initial state. 4

${ }^{3}$ In Sec. III C of Ref. [26], Caves uses his path-integral formulation of measurement theory to analyze measurements of the discrete second time derivative of the position of a free particle on which a classical force acts. His analysis reveals the same conclusion as we obtain in our pedagogical example: the measured quantity contains information about the force and is devoid of any influence from the particle's initial state.

${ }^{4}$ The crucial idea of avoiding the influence of the test-mass initial state by monitoring differences of observables $\left[\left(\hat{Q}_{2}-\hat{Q}_{1}\right)-\left(\hat{Q}_{1}\right.\right.$ $-\hat{Q}_{0}$ ) in our case] is contained in a paper and book by Alter and Yamamoto $[33,34]$. Alter and Yamamoto point out that, for a test mass on which a classical force acts, the momentum $\hat{p}(t)$ at time $t$ and the momentum $\hat{p}(0)$ at time 0 are correlated in that $\hat{p}(t)$ $=\hat{p}(0)+\int_{0}^{t} d t^{\prime} F\left(t^{\prime}\right) ; \quad$ so, if one measures $\hat{p}(t)-\hat{p}(0)$ $=\int_{0}^{t} d t^{\prime} F\left(t^{\prime}\right)$, one thereby can get information about the force without any contaminating influence of the test-mass initial state. They say (p. 96 of [34]) that this is so not only when one measures directly the difference $\hat{p}(t)-\hat{p}(0)$ (as in Sec. 7.2.2 of their [34]), but also when the difference is determined computationally from the results of measurements of $\hat{p}(t)$ and $\hat{p}(0)$ [an analog of our way of monitoring $\left.\left(\hat{Q}_{2}-\hat{Q}_{1}\right)-\left(\hat{Q}_{1}-\hat{Q}_{0}\right)\right]$. When going on to discuss position measurements, Alter and Yamamoto note that $\hat{x}(t)-\hat{x}(0)$ $=\hat{p}(0) t / m+\int_{0}^{t} d t^{\prime} \int_{0}^{t^{\prime}} d t^{\prime \prime} F\left(t^{\prime \prime}\right) / m$, so a measurement of $\hat{x}(t)$ $-\hat{x}(0)$ is contaminated [via $\hat{p}(0) t / m$ ] by noise from the test-mass initial state. Examining this contamination, they conclude that "force detection via position monitoring of a free mass is limited by 
These statistical properties are embodied in the means, over all the experiments, of arbitrary functions $G(\widetilde{R})$. The theory of measurement tells us that, because the $\hat{Q}$ 's all commute, the computed mean of $G(\widetilde{R})$ is given by

$$
\text { [computed mean of } G(\widetilde{R})]=\langle\operatorname{in}|G(\hat{R})| \text { in }\rangle,
$$

where $\hat{R}$ is the operator corresponding to $\widetilde{R}$

$$
\begin{aligned}
\hat{R} & =\hat{Q}_{0}-2 \hat{Q}_{1}+\hat{Q}_{2} \\
& =-\left(\xi_{0}-2 \xi_{1}+\xi_{2}\right)+\left[\hat{Q}_{0}^{\text {before }}-2 \hat{Q}_{1}^{\text {before }}-\frac{\hat{P}_{1} \tau}{\mu}+\hat{Q}_{2}^{\text {before }}\right]
\end{aligned}
$$

cf. Eq. (2.17). Because $\hat{R}$ is independent of $\hat{x}_{o}$ and $\hat{p}_{o}$, the computed mean (2.22) and thence all the measurement statistics of $\widetilde{R}$ will be completely independent of the test-mass quantum mechanics, and in particular independent of the test mass's initial state. Moreover, Eq. (2.22) implies that, so far as measurement results and statistics are concerned, measuring the $\hat{Q}$ 's and then computing $\widetilde{R}$ is completely equivalent to measuring $\hat{R}$ directly.

Although $\hat{R}$ is independent of $\hat{x}_{o}$ and $\hat{p}_{o}$ it contains

$$
\xi_{0}-2 \xi_{1}+\xi_{2}=\frac{1}{\mu} \int_{0}^{2 \tau}(\tau-|t-\tau|) F(t) d t \equiv \frac{\tau^{2}}{\mu} \bar{F},
$$

where $\bar{F}$ is a weighted mean of the classical force $F$ over the time interval $0<t<2 \tau$; cf. Eq. (2.16). ${ }^{5}$ Thus, this measurement of $\hat{R}$ is actually a measurement of $\bar{F}$, and is contaminated by quantum noise from the meters but not by quantum noise from the test mass. The only role of the quantum mechanical test mass is to feed the classical signal $\bar{F}$ and the meter back-action noise $\hat{P}_{1} \tau / m$ into the output.

For those readers who are uncomfortable with our use of the Heisenberg picture to derive this very important result, we present a Schrödinger-picture derivation in Appendix A.

... the SQL" [33]. While this conclusion is correct when one monitors $\hat{x}(t)-\hat{x}(0)$ in the manner envisioned by Alter and Yamamoto, it is incorrect for the alternative strategy embodied in our model problem. Instead of monitoring $\hat{x}(t)-\hat{x}(0)$, one should monitor $\hat{x}(0)-2 \hat{x}(t)+\hat{x}(2 t)$, which for a free mass is independent of both $\hat{x}_{o} \equiv \hat{x}(0)$ and $\hat{p}_{o} \equiv \hat{p}(0)$. Then the measurement output contains information about the force $F(t)$, uncontaminated by any influence of the test-mass initial state.

${ }^{5}$ Notice that, aside from meter noise, $\xi_{r}$ is equal to $\hat{x}\left(t_{r}\right)$ $-\hat{p}(0) t_{r} / \mu$ [Eq. (2.15)], which is a QND observable (as M.B. Mensky pointed out long ago). Therefore, the quantity $\hat{R}$ that we measure can be regarded as a discrete second time derivative of a QND observable-which suggests that it can be the foundation for a QND measurement; see Sec. II B 5 below.
This three-meter thought experiment is a prototype for our discussion of gravitational-wave interferometers in Sec. III B. There as here, the linearity of the output in the testmass initial positions and momenta will enable us to find a linear signal processing procedure that removes the initialstate influence. Here that procedure was a discrete second time derivative. For an interferometer it will be a discrete Fourier transform of the measured photon flux (the output), and a discarding of Fourier components at the test masses' natural frequencies (the $1 \mathrm{~Hz}$ pendular swinging frequency in the case of conventional interferometers).

For an elegant path-integral analysis of the removal of test-mass initial conditions from the output of measurements of any harmonic oscillator on which a classical force acts, see the last portion of Sec. III C of Caves [26].

\section{The SQL for the classical-force measurement}

How small can the test-mass noise be? A "naive" optimization of the meters leads to the standard quantum limit on the measured force, in the same way as a "naive" optimization of a gravitational-wave interferometer's design (forcing it to retain the conventional LIGO-I optical topology but optimizing its laser power) leads to the gravitational-wave SQL. Specifically:

Let the three meters all be prepared in initial states that are "naive" in the sense that they have no correlations between their coordinates and momenta. Then Eqs. (2.23) and (2.24) imply that the variance of the measured mean force is

$$
\begin{aligned}
(\Delta \bar{F})^{2}= & \frac{\mu^{2}}{\tau^{4}}\left[\left(\Delta Q_{0}^{\text {before }}\right)^{2}+\left(2 \Delta Q_{1}^{\text {before }}\right)^{2}\right. \\
& \left.+\left(\frac{\Delta P_{1} \tau}{\mu}\right)^{2}+\left(\Delta Q_{2}^{\text {before }}\right)^{2}\right] .
\end{aligned}
$$

Obviously, this variance is minimized by putting meters 0 and 2 into (near) eigenstates of their coordinates, so $\Delta Q_{0}^{\text {before }}=\Delta Q_{2}^{\text {before }}=0$. To minimize the noise from meter 1 , we require that it have the smallest variances compatible with its uncertainty relation,

$$
\Delta Q_{1}^{\text {before }} \Delta P_{1}=\frac{\hbar}{2},
$$

and we adjust the ratio $\Delta Q_{1}^{\text {before }} / \Delta P_{1}$ so as to minimize $(\Delta \bar{F})^{2}$. The result is

$$
(\Delta \bar{F})^{2}=\frac{2 \mu \hbar}{\tau^{3}},
$$

which is the SQL for measuring a classical force, up to a factor of order unity; cf. Sec. 8.1 of Ref. [3].

It is evident from this analysis that the true physical origin of the SQL in classical force measurements is the meter's noise, not the test-mass noise. On the other hand, the quantum properties of the meter and of the test mass are intimately coupled through the requirement that the meter commutators cancel the test-mass commutator in the measurement output, so that $\left[\hat{Q}_{r}, \hat{Q}_{s}\right]=0$ [Eq. (2.18)]. This 
intimate coupling - which, as we have discussed, has enormous generality-ensures that the SQL can be derived equally well from test-mass considerations and from meter considerations. We saw this explicitly in Sec. I for an interferometric gravitational-wave detector.

\section{Beating the $S Q L$}

Equation (2.23) suggests a way to beat the classical-force SQL and, in fact, achieve arbitrarily high accuracy: As in our "naive" optimization, before the measurement we place meters 0 and 2 in (near) eigenstates of their coordinates, so $\Delta Q_{0}=\Delta Q_{2}=0$, but instead of putting meter 1 in a "naive" state with uncorrelated coordinate and momentum, we place it in a (near) eigenstate of

$$
\hat{Q}_{1}^{\text {squeeze }} \equiv \hat{Q}_{1}^{\text {before }}-\hat{P}_{1} \tau / 2 \mu
$$

(This meter-1 state is analogous to the squeezed-vacuum state, which Unruh [18] has proposed be inserted into a conventional interferometer's dark port in order to beat the gravitational-wave SQL; see Sec. II C below.) These initial meter states, together with Eqs. (2.23) and (2.22), guarantee that the variance of the computed quantity $\widetilde{R}$ vanishes $\Delta \widetilde{R}$ $=0$, and thence [via Eqs. (2.24) and (2.23)] that the variance of the measured mean force vanishes, $\Delta \bar{F}=0$. Thus, by putting the initial state of meter 1 into the analog of a squeezed vacuum state, we can achieve an arbitrarily accurate measurement of $\bar{F}$.

The SQL can also be evaded by modifying the meters' measured quantities instead of modifying their initial states. Specifically, measure $\hat{Q}_{0}$ and $\hat{Q}_{2}$ as before, but on meter 1 instead of measuring the coordinate $\hat{Q}_{1}$, measure the following linear combination of the coordinate and momentum (with the coefficient $\alpha$ to be chosen below):

$$
\begin{aligned}
\hat{Q}_{1}^{\mathrm{var}} & =\hat{Q}_{1}+\alpha \hat{P}_{1} \\
& =\hat{Q}_{1}^{\text {before }}-\hat{x}_{0}-\frac{\hat{p}_{0}}{\mu} \tau-\frac{\hat{P}_{0}}{\mu} \tau-\alpha \hat{P}_{1}-\xi_{1} .
\end{aligned}
$$

From Eqs. (2.29), (2.17) and (2.18), we see that the output observables $\left\{\hat{Q}_{0}, \hat{Q}_{1}^{\mathrm{var}}, \hat{Q}_{2}\right\}$ all commute with each other. Therefore, when we combine their measured values into the discrete second time derivative

$$
\widetilde{R}_{\mathrm{var}} \equiv \widetilde{Q}_{0}-2 \widetilde{Q}_{1}^{\mathrm{var}}+\widetilde{Q}_{2},
$$

its statistics will be the same as if we had directly measured the corresponding operator

$$
\begin{aligned}
\hat{R}_{\mathrm{var}}= & \widetilde{Q}_{0}-2 \widetilde{Q}_{1}^{\mathrm{var}}+\widetilde{Q}_{2} \\
= & -\left(\xi_{0}-2 \xi_{1}+\xi_{2}\right)+\left[\hat{Q}_{0}^{\text {before }}-2 \hat{Q}_{1}^{\text {before }}\right. \\
& \left.+\frac{\hat{P}_{1}}{\mu} \tau-2 \alpha \hat{P}_{1}+\hat{Q}_{2}^{\text {before }}\right] .
\end{aligned}
$$

Evidently, we should choose $2 \alpha=\tau / \mu$, so the quantity measured is

$$
\hat{Q}_{1}^{\mathrm{var}}=\hat{Q}_{1}+\hat{P}_{1} \frac{\tau}{2 \mu}
$$

Then Eqs. (2.31) and (2.24) imply that

$$
\hat{R}_{\mathrm{var}}=-\frac{\tau^{2}}{\mu} \bar{F}+\hat{Q}_{0}^{\text {before }}-2 \hat{Q}_{1}^{\text {before }}+\hat{Q}_{2}^{\text {before }}
$$

Therefore, by measuring our chosen linear combination of meter 1 's coordinate and momentum, and then computing the discrete second time derivative, we have succeeded in removing from our output observable $\hat{R}_{\mathrm{var}}$ not only the test-mass variables $\hat{x}_{o}, \hat{p}_{o}$, but also the back-action influence of the meters on the measurement (all three $\hat{P}_{r}$ 's). Correspondingly, by putting the meters into "naive" initial states (states with no position-momentum correlations) that are near eigenstates of their coordinates (so $\Delta Q_{0}, \Delta Q_{1}, \Delta Q_{2}$ are arbitrarily small and the back-action fluctuations $\Delta P_{0}, \Delta P_{1}, \Delta P_{2}$ are arbitrarily large), then from the computed quantity $\widetilde{R}_{\text {var }}$, we can infer the mean position $\bar{F}$ with arbitrarily good precision.

This strategy was devised, in the context of optical measurements of test masses, by Vyatchanin, Matsko and Zubova [6-9], and is called a quantum variational measurement. A gravitational-wave interferometer that utilizes it (and can beat the SQL) is called a variational output interferometer [13].

Of course, one can also beat the SQL for force measurements by a combination of putting the meters into initially squeezed states and performing a quantum variational measurement on their outputs. A gravitational-wave detector based on this mixed strategy is called a squeezed variational interferometer, and may have practical advantages over squeezed-input and variational-output interferometers [13].

\section{Pulsed-light measurements of test-mass position}

Our two pedagogical examples (single position measurement, Sec. II A, and classical force measurement, Sec. II B) can be realized using pulsed-light measurements of the testmass position. We exhibit this realization in part to lend reality to our highly idealized examples, and in part as a bridge from those simple examples to gravitational-wave interferometers with their far greater complexity (Sec. III below).

In each pulsed-light measurement we reflect a laser light pulse, with carrier frequency $\omega_{o}$ and Gaussian-profile duration $\tau_{o}$, off a mirror on the front face of the test mass, and from the light's phase change we deduce the test-mass position $\hat{x}$ averaged over the pulse. This is a concrete realization not only of the pulsed measurements of our pedagogical examples, but also of a Heisenberg microscope. We presume that the pulse duration $\tau_{o}$ is long compared to the light's period $2 \pi / \omega_{o}$, but short compared to the time $\tau$ between measurements. 
We shall analyze in detail one such pulsed measurement. The electric field of the reflected wave, at some fiducial location, is

$$
\begin{aligned}
\hat{E}(t)= & \sqrt{\frac{2 \pi \hbar \omega_{0}}{c S}}\left(e ^ { - i \omega _ { 0 } t } \left[A_{0} e^{-t^{2} / 2 \tau_{0}^{2}}\left(1+\frac{2 i \omega_{0}}{c} \hat{x}(t)\right)\right.\right. \\
& +\hat{a}(t)]+ \text { H.c. })
\end{aligned}
$$

where $A_{0}$ is the pulse's amplitude, $S$ is its cross sectional area, $c$ is the speed of light, $2\left(\omega_{0} / c\right) \hat{x}(t)$ is the phase shift induced by the test-mass displacement $\hat{x}(t)$, "H.c." means Hermitian conjugate, and $\hat{a}(t)$ is the electric field's amplitude operator. Because we are concerned only about timescales of order the pulse duration $\tau_{0}$ or longer, which means side-band frequencies $\lesssim 1 / \tau_{0} \ll \omega_{0}$, we can use the quasimonochromatic approximation to the commutation relation for $\hat{a}(t)[35]$ :

$$
\left[\hat{a}(t), \hat{a}^{\dagger}\left(t^{\prime}\right)\right]=\delta\left(t-t^{\prime}\right) .
$$

Note that, when decomposed into quadratures with respect to the carrier frequency, this electric field is

$$
\hat{E}(t)=\hat{E}_{A}(t) \cos \omega_{o} t+\hat{E}_{\phi}(t) \sin \omega_{o} t,
$$

where $\hat{E}_{A}$ and $\hat{E}_{\phi}$, the amplitude and phase quadratures (i.e., the quadrature components oriented along and perpendicular to the amplitude direction in the quadrature plane) are given by

$$
\begin{aligned}
& \hat{E}_{A}=2 \sqrt{\frac{2 \pi \hbar \omega_{o}}{c S}}\left[A_{o} e^{\left.-t^{2} / 2 \tau_{o}^{2}+\left(\frac{\hat{a}(t)+\hat{a}^{\dagger}(t)}{2}\right)\right],}\right. \\
& \hat{E}_{\phi}=2 \sqrt{\frac{2 \pi \hbar \omega_{o}}{c S}}\left[2 A_{o} \frac{\omega_{o}}{c} e^{\left.-t^{2} / 2 \tau_{o x}^{2} \hat{x}(t)+\left(\frac{\hat{a}(t)-\hat{a}^{\dagger}(t)}{2 i}\right)\right] .}\right.
\end{aligned}
$$

The power $\hat{W}(t)$ in the incident wave can be written as the sum of a mean power $\langle W(t)\rangle$ and a fluctuating (noise) part $\widetilde{W}(t)$ :

$$
\begin{aligned}
\hat{W}(t) & =S c \frac{\overline{\hat{E}^{2}(t)}}{4 \pi}=\langle W(t)\rangle+\widetilde{W}(t), \\
\langle W(t)\rangle & =\hbar \omega_{0} A_{0}^{2} e^{-t^{2} / \tau_{0}^{2},} \\
\widetilde{W}(t) & =2 \hbar \omega_{0} A_{0} e^{-t^{2} / 2 \tau_{0}^{2}}\left(\frac{\hat{a}(t)+\hat{a}^{\dagger}(t)}{2}\right) .
\end{aligned}
$$

Here the over bar means "average over the carrier period." The light-pressure force on the mirror is $\hat{F}(t)=2 \hat{W}(t) / c$. The fluctuating part of this, $\widetilde{F}(t)=2 \widetilde{W}(t) / c$, is the backaction of the measurement on the test mass, and it produces the back-action momentum change

$$
\begin{aligned}
\delta \hat{p}_{\mathrm{BA}} & =\int_{-\infty}^{\infty} d t \frac{2 \widetilde{W}(t)}{c} \\
& =\frac{4 \hbar \omega_{0}}{c} A_{0} \int_{-\infty}^{\infty} d t e^{-t^{2} / 2 \tau_{0}^{2}}\left(\frac{\hat{a}(t)+\hat{a}^{\dagger}(t)}{2}\right) .
\end{aligned}
$$

The test-mass momentum before and after the pulsed measurement are related by

$$
\hat{p}_{\text {after }}=\hat{p}_{\text {before }}+\delta \hat{p}_{\text {BA }} .
$$

The experimenter deduces the phase shift $\left(2 \omega_{o} / c\right) \hat{x}(t)$ and thence the test-mass displacement $\hat{x}(t)$ by measuring the electric field's phase quadrature $\hat{E}_{\phi}$ (e.g., via interferometry or homodyne detection). More precisely, the experimenter measures the phase quadrature integrated over the pulse, obtaining a result proportional to

$$
\begin{aligned}
\hat{x}_{\text {meas }} & =\sqrt{\frac{c S}{2 \hbar \omega_{o}}} \frac{c}{4 \pi \omega_{0} \tau_{0} A_{0}} \int_{-\infty}^{+\infty} e^{-t^{2} / 2 \tau_{0}^{2} \hat{E}_{\phi}(t) d t} \\
& =\hat{x}+\delta \hat{x}_{\text {meas }}
\end{aligned}
$$

cf. Eq. (2.37b). Here $\hat{x}$ is the mirror position averaged over the short pulse, $\hat{x}_{\text {meas }}$ is the measured value of $\hat{x}$, and $\delta \hat{x}_{\text {meas }}$ is the measurement noise superposed on the output by the light pulse

$$
\delta \hat{x}_{\text {meas }}=\frac{c}{2 \sqrt{\pi} \omega_{0} \tau_{0} A_{0}} \int_{-\infty}^{\infty} d t e^{-t^{2} / 2 \tau_{0}^{2}}\left(\frac{\hat{a}(t)-\hat{a}^{\dagger}(t)}{2 i}\right) .
$$

It is straightforward, from the commutator $\left[\hat{a}(t), \hat{a}^{\dagger}\left(t^{\prime}\right)\right]$ $=\delta\left(t-t^{\prime}\right)$, to show that the measurement noise and the back-action impulse have the same commutator

$$
\left[\delta \hat{x}_{\text {meas }}, \delta \hat{p}_{\mathrm{BA}}\right]=-i \hbar
$$

as for the idealized single measurement of Sec. II A [Eq. (2.7)], and correspondingly the mirror's measured position and its final momentum commute,

$$
\left[\hat{x}_{\text {meas }}, \hat{p}_{\text {after }}\right]=0 .
$$

The fundamental equations (2.41), (2.40), (2.43) and (2.44) for this pulsed-light measurement are the same as those Eqs. (2.4), (2.7), (2.8) for our idealized single measurement, and this measurement is thus a realistic variant of the idealized one. Similarly, a sequence of pulsed-light measurements can be used to monitor a classical force acting on a mirror, and the fundamental equations for such measurements are the same as for the idealized example of Sec. II B.

In such pulsed-light experiments, the measurement noise $\delta \hat{x}_{\text {meas }}$ is proportional to the fluctuations of the light's phase quadrature $\hat{E}_{\phi}$ [Eqs. (2.37b) and (2.42)], and the back-action impulse $\delta \hat{p}_{\mathrm{BA}}$ is proportional to the fluctuations of its ampli- 
tude quadrature $\hat{E}_{A}$ [Eqs. (2.37a) and (2.39)]. Of course, experimenters can measure any quadrature of the reflected light pulse that they wish. To achieve a QND quantum variational measurement of a classical force acting on the test mass [6-9], the experimenter should measure $\hat{Q}_{1}^{\mathrm{var}}=\hat{Q}_{1}$ $+\hat{P}_{1} \tau / 2 \mu$ in the language of our idealized thought experiment [Eq. (2.32)], which [by Eqs. (2.5)] translates into $-\delta \hat{x}_{\text {meas }}+\delta \hat{p}_{\mathrm{BA}} \tau / 2 \mu$ plus the light's signal and carrier, which in turn is a specific linear combination of the light's amplitude and phase quadratures $\hat{E}_{A}$ and $\hat{E}_{\phi}$ [Eqs. (2.37), (2.42), (2.39)]. The experimenter can also prepare the incident pulse in a squeezed state, in the manner required for an Unruh-type [18] QND measurement of the classical force. In the language of our idealized thought experiment, the desired squeezed state is a (near) eigenstate of $\hat{Q}_{1}^{\text {squeeze }}=\hat{Q}_{1}$ $-\hat{P}_{1} \tau / 2 \mu$ [Eq. (2.28)], which translates into a near eigenstate of $\delta \hat{x}_{\text {meas }}+\delta \hat{p}_{\mathrm{BA}} \tau / 2 \mu$ [cf. Eqs. (2.5)], or equivalently a near eigenstate of a specific linear combination of $\hat{E}_{A}$ and $\hat{E}_{\phi}$.

\section{GRAVITATIONAL-WAVE INTERFEROMETERS AND OTHER PHOTODETECTION-BASED DEVICES}

We now turn our attention to gravitational-wave interferometers and other real, high-precision devices for monitoring classical forces that act on test masses. Our goal is to prove that for these devices, as for our idealized examples, the force-measurement precision can be made completely independent of the test mass's quantum properties, including its initial state and that this can be achieved by an appropriate filtering of the output data stream.

As in our examples, this conclusion relies on the vanishing commutator of the observables that constitute the output data stream. We shall now discuss the nature of the output data stream and show that its commutator does, indeed, vanish.

\section{A. Vanishing commutator of the output}

For interferometers and many other force-monitoring devices, the data stream, shortly before amplification to classical size, is encoded in an output light beam, and that beam is sent into a photodetector which monitors its photon number flux $\hat{\mathcal{N}}(t)$. The photodetector and associated electronics integrate up $\hat{\mathcal{N}}(t)$ over time intervals with duration $\tau$ long compared to the light beam's carrier period, $\tau \gg 2 \pi / \omega_{o}$ $\sim 10^{-15} \mathrm{~s}$, but short compared to the shortest timescales on which the classical force changes $\left(\tau \ll \tau_{\mathrm{GW}} \sim 10^{-3} \mathrm{~s}\right.$ for the gravitational waves sought by interferometers). For LIGO-I interferometers, the integration time has been chosen to be $\tau=5 \times 10^{-5} \mathrm{~s}$. The result is a discretized output data stream, whose Hermitian observables are the numbers of photons in the successive data samples,

$$
\hat{N}_{j}=\int_{-\infty}^{\infty} s\left(t-t_{j}\right) \hat{\mathcal{N}}(t) d t
$$

Here $t_{j}=j \tau_{0}$ is the time of sample $j$, and $s(t)$ is a sampling function approximately equal to unity during a time interval $\Delta t=\tau_{0}$ centered on $t_{j}$ and zero outside that time interval.

The photon number samples $\hat{N}_{j}$ are the analogs, for an interferometer or other force-monitoring device, of the meter coordinates $\hat{Q}_{j}$ in the idealized example of Sec. II B.

In Appendix $\mathrm{C}$ we show that for any free light beam, the number flux operator, evaluated at a fixed plane orthogonal to the optic axis (e.g. at the entrance to the photodetector) self commutes,

$$
\left[\hat{\mathcal{N}}(t), \hat{\mathcal{N}}\left(t^{\prime}\right)\right]=0
$$

This guarantees, in turn, that all the output photon-number data samples (3.1) commute with each other

$$
\left[\hat{N}_{j}, \hat{N}_{k}\right]=0 \text {. }
$$

As we shall see below [Eq. (3.9)], the initial position and momentum of the test mass, $\hat{x}_{o}$ and $\hat{p}_{o}$, appear linearly in the output variables $\hat{\mathcal{N}}(t)$ and $\hat{N}_{j}$. They obviously will produce nonzero contributions to the output commutators. As in our simple examples (Sec. II), these nonzero test-mass contributions must be canceled by identical nonzero contributions from noncommutation of the measurement noise (photon shot noise) and the back-action noise (radiation-pressure noise).

\section{B. Devising a filter to remove test-mass quantum noise}

The vanishing output commutators constitute our first underpinning for freeing the measurements from the influence of test-mass quantization. As in the idealized measurements of Sec. II B, the vanishing commutators guarantee a key property of the data analysis: If, from each specific realization of the output data stream $\left\{\widetilde{N}_{1}, \widetilde{N}_{2}, \ldots\right\}$, our data analysis produces a new set of quantities (the "filtered output variables")

$$
\widetilde{R}_{J}\left(\widetilde{N}_{1}, \widetilde{N}_{2}, \ldots\right)
$$

then the statistics of these $\widetilde{R}_{J}$ will be identically the same as if we had directly measured the corresponding observables

$$
\hat{R}_{J}\left(\hat{N}_{1}, \hat{N}_{2}, \ldots\right)
$$

rather than computing them from the measured $\tilde{N}_{j}$ 's. Therefore, we can regard our interferometer (or other device) as measuring the filtered output observables $\left\{\hat{R}_{1}, \hat{R}_{2}, \ldots\right\}$, whatever those observables may be.

By analyzing the test-mass dynamics of the interferometer (or other measuring device) in the Heisenberg picture, one can learn how the test-mass initial position $\hat{x}_{o}$ and momentum $\hat{p}_{o}$ influence the operators $\left\{\hat{N}_{1}, \hat{N}_{2}, \ldots\right\}$. One can then deduce a set of filtered observables $\left\{\hat{R}_{1}, \hat{R}_{2}, \ldots\right\}$ in which $\hat{x}_{o}$ and $\hat{p}_{o}$ do not appear but the gravitational-wave or other classical force information is retained. (These will be the analogues of $\hat{R}=\hat{Q}_{0}-2 \hat{Q}_{1}+\hat{Q}_{2}$ [Eq. (2.23)] in our simple 
model problem.) The filter that leads from $\left\{\hat{N}_{1}, \hat{N}_{2}, \ldots\right\}$ to $\left\{\hat{R}_{1}, \hat{R}_{2}, \ldots\right\}$, when applied to the output (c-number) data $\left\{\widetilde{N}_{1}, \widetilde{N}_{2}, \ldots\right\}$ to produce $\left\{\widetilde{R}_{1}, \widetilde{R}_{2}, \ldots\right\}$, is guaranteed to remove all influence of $\hat{x}_{o}$ and $\hat{p}_{o}$, and thence all influence of the test-mass initial state.

\section{Influence of $\hat{x}_{o}$ and $\hat{p}_{o}$ on the output data}

To make this more specific, let us explore how $\hat{x}_{o}$ and $\hat{p}_{o}$ influence the output data train.

To very high accuracy (sufficient for our purposes), interferometers (and most other force-measuring devices) are linear. The inputs are: (i) the test-mass position $\hat{x}(t)$ [actually, the difference between four test-mass positions in the case of an interferometer; Eq. (1.2)], and (ii) the electric field operators $\hat{E}_{a}(t), a=1,2, \ldots$ for the field fluctuations that enter the interferometer at the bright port, at the dark port, and at all light-dissipation locations (e.g., at mirrors where bits of light scatter out of the optical train and reciprocally new bits of field fluctuations scatter into it); see, e.g., the detailed analysis of interferometers in Ref. [13]. The output photon flux is a linear functional of these inputs,

$$
\hat{\mathcal{N}}(t)=\int_{-\infty}^{t}\left[K_{x}\left(t-t^{\prime}\right) \hat{x}\left(t^{\prime}\right)+\sum_{a} K_{a}\left(t-t^{\prime}\right) \hat{E}_{a}\left(t^{\prime}\right)\right] d t^{\prime} ;
$$

cf. the discussion in Appendix C. The $\hat{E}_{a}$ terms constitute the photon shot noise (analogs of $\hat{Q}_{r}^{\text {before }}$ in our idealized example, Sec. II B).

The test-mass initial observables $\hat{x}_{o}$ and $\hat{p}_{o}$ enter $\hat{\mathcal{N}}(t)$ and thence $\left\{\hat{N}_{1}, \hat{N}_{2}, \ldots\right\}$, through $\hat{x}(t)$ in a manner governed by the test masses' free dynamics. The nature of that free dynamics depends on the interferometer design. We shall consider two examples in turn: interferometers with pendular dynamics, and signal-recycled interferometers. These examples should be easily extendable to any other type of interferometer than might be conceived in the future.

\section{Interferometers with pendular dynamics}

In conventional gravitational-wave interferometers (e.g. LIGO-I, VIRGO and TAMA) and in the QND interferometers analyzed by Kimble et al. [13], the test masses swing sinusoidally at $\sim 1 \mathrm{~Hz}$ frequency in response to their suspensions' pendular restoring force (as modified slightly by the optical cavities' radiation-pressure force):

$$
\hat{x}_{\text {free }}(t)=\hat{x}_{o} \cos \omega_{m} t+\frac{\hat{p}_{o}}{\mu \omega_{m}} \sin \omega_{m} t
$$

Here $\mu$ is the reduced mass $(1 / 4$ the actual mass of one test mass in the case of an interferometer) and $\omega_{m} \sim 2 \pi \times 1 \mathrm{~Hz}$ is the pendular swinging frequency. There is no significant damping of the free motion (3.7) because the experimenters take great pains to liberate the test masses from all damping; the typical damping times in LIGO-I are of order a day, and in advanced interferometers (LIGO-II and beyond) will be of order a year or more [14,36], which is far longer than the data segments used in the data analysis.

Superimposed on the free test-mass dynamics (3.7) are (i) the influence $\xi_{\mathrm{GW}}(t)$ of the gravitational-wave signal, (ii) the "back-action" influence $\hat{x}_{\mathrm{BA}}(t)$ of the light's fluctuating radiation pressure (which is linear in the input fields $\hat{E}_{a}$ and is the analog of the $\hat{P}_{r}$ and $\delta p_{\mathrm{BA}}$ of our discrete model problems), and (iii) the influence $\xi_{\text {other }}(t)$ of a variety of other forces-low-frequency feedback forces from servo systems, thermal-noise forces, seismic vibration forces, etc:

$$
\hat{x}(t)=\hat{x}_{\text {free }}(t)+\xi_{\mathrm{GW}}(t)+\hat{x}_{\mathrm{BA}}(t)+\xi_{\text {other }}(t) .
$$

Inserting Eq. (3.7) into Eq. (3.8) and then Eq. (3.8) into Eq. (3.6) we see that, for a test-mass with pendular dynamics, the initial test-mass position and momentum operators appear in the output flux operator in the form

$$
\begin{aligned}
\hat{\mathcal{N}}(t)= & \int_{-\infty}^{t} K_{x}\left(t-t^{\prime}\right)\left[\hat{x}_{o} \cos \omega_{m} t^{\prime}+\frac{\hat{p}_{o}}{\mu \omega_{m}} \sin \omega_{m} t^{\prime}\right] d t^{\prime} \\
& + \text { (other contributions) }
\end{aligned}
$$

The interferometer's transfer function $K_{x}\left(t-t^{\prime}\right)$ is independent of absolute time and thus transforms frequency- $\omega_{m}$ inputs into frequency- $\omega_{m}$ outputs. Therefore, $\hat{x}_{o}$ and $\hat{p}_{o}$ appear in the output solely at frequency $\omega_{m} / 2 \pi \sim 1 \mathrm{~Hz}$. Now, because the output data generally have large noise (seismic and other) at frequencies below $\sim 10 \mathrm{~Hz}$, it is routine, in interferometers, to high-pass filter the output data so as to remove frequencies below $\sim 10 \mathrm{~Hz}$. When one does so, one automatically removes all influence of $\hat{x}_{o}$ and $\hat{p}_{o}$ from the filtered data $\widetilde{R}_{J}$ [Eq. (3.4)]. This is a precise analog of applying the discrete second time derivative to the output data in our simple example (Sec. II B) so as to remove $\hat{x}_{o}$ and $\hat{p}_{o}$ from the data; and it is a realization of a general class of measurement procedures, for a harmonic oscillator on which a classical force acts, that is analyzed by Caves using his path integral formalism (last part of Sec. III C of Ref. [26]).

\section{Signal-recycled interferometers}

A signal-recycling mirror, placed at an interferometer's output port, sends information about the test-mass position $\hat{x}(t)$ back into the interferometer as part of the back-action (radiation-pressure) force, and thereby alters the free testmass dynamics. The altered free dynamics have been analyzed in detail by Buonanno and Chen [17]; they find that the test masses and the interferometer's side-band light form a coupled system with four degrees of freedom, so $\hat{x}_{o}$ and $\hat{p}_{o}$ appear in $\hat{x}_{\text {free }}(t)$, and thence in $\hat{x}(t)$ and thence in $\hat{\mathcal{N}}(t)$ at four discrete frequencies $\omega_{A}(A=1,2,3,4)$. Correspondingly, in the output data train, the influence of the test-mass initial state is confined to the Fourier components at the frequencies $\omega_{A}$.

If these frequencies were real, then one could remove the influence of the test-mass initial state from the data by filtering out the data's Fourier components at these four frequen- 
cies. However, as Buonanno and Chen [17] discuss, such filtering is not necessary: The frequencies are actually complex with imaginary parts that produce damping on time scales $\leqslant 1$ second (when a servo is introduced to control an instability). Therefore, the influence of $\hat{x}_{o}$ and $\hat{p}_{o}$ on the output flux operator $\hat{\mathcal{N}}(t)$ damps out quickly, and correspondingly (see the end of Sec. III A), the influence of the testmass initial state on the output data train damps out quickly without any filtering.

\section{CONCLUSIONS}

To reiterate: In an interferometer (and many other forcemeasuring devices), the output signal is encoded in the photon number flux operator $\hat{\mathcal{N}}(t)$ of a light beam, which is converted into discrete photon number samples $\hat{N}_{j}$ by a photodetector and electronics. These outputs have vanishing commutators $\left[\hat{\mathcal{N}}(t), \hat{\mathcal{N}}\left(t^{\prime}\right)\right]=0$ and $\left[\hat{N}_{j}, \hat{N}_{k}\right]=0$ and thus can be thought of as classical quantities. These outputs are linear in the initial test-mass position $\hat{x}_{o}$ and momentum $\hat{p}_{o}$ and involve no other test-mass variables. The output commutators manage to vanish because the photon back-action noise and photon shot noise have commutators that cancel those of $\hat{x}_{o}$ and $\hat{p}_{o}$.

In the output $\hat{\mathcal{N}}(t)$ of any interferometer with pendular dynamics, $\hat{x}_{o}$ and $\hat{p}_{o}$ appear only at the pendular frequency $\omega_{m} / 2 \pi \sim 1 \mathrm{~Hz}$, and all influences of $\hat{x}_{o}$ and $\hat{p}_{o}$ (including all influences of the test-mass initial state) are removed completely from the data by the high-pass filtering that is routine for interferometers. For other types of interferometers, with different test-mass dynamics, other data filtering procedures will remove the influence of $\hat{x}_{o}$ and $\hat{p}_{o}$ and the test-mass initial state-and in some cases (e.g., a signal-recycled interferometer) no filtering is needed at all.

This complete removal of all influence of $\hat{x}_{o}$ and $\hat{p}_{o}$ from the filtered data implies the answers to the three questions posed in the introduction of this paper (Sec. I): (i) The testmass quantum mechanics has no influence on the interferometer's noise; the only quantum noise is that arising from the light. (ii) Therefore, when analyzing a candidate interferometer design, one need not worry about the test-mass quantum mechanics, except for using it to feed the gravity-wave signal and the back-action noise through the test mass to the photon-flux output. (iii) Similarly, when conceiving new designs for interferometers, one need not worry about the testmass quantum mechanics-except for devising appropriate data filters to remove $\hat{x}_{o}$ and $\hat{p}_{o}$ from the data.

\section{ACKNOWLEDGMENTS}

For helpful advice or email correspondence, we thank Orly Alter, Alessandra Buonanno, Carlton Caves, Yanbei Chen, Crispin Gardiner, William Unruh, Yoshihisa Yamamoto, and the members of the 1998-1999 Caltech QND Reading Group, most especially Constantin Brif, Bill Kells, Jeff Kimble, Yuri Levin and John Preskill. This research was supported in part by NSF grants PHY-9503642, PHY-
9900776, PHY-0098715, and PHY-0099568, by the Russian Foundation for Fundamental Research grants No. 96-0216319a and No. 97-02-0421g, and (for V.B.B., F.Ya.K. and S.P.V.) by the NSF through Caltech's Institute for Quantum Information.

\section{APPENDIX A: TRIPLE MEASUREMENT IN THE SCHRÖDINGER PICTURE}

In this appendix we present a Schrödinger-picture analysis of the most important of this paper's pedagogical thought experiments (Sec. II B): a triple measurement of the position of a free test mass, using three independent meters, with the goal of determining the mean classical force $\bar{F}$ acting on the test mass without any contaminating noise whatsoever from the test mass's initial state. Our analysis will proceed in three steps: (i) an analysis of one of the position measurements (any one of the three), Appendix A 1; (ii) [relying on step (i)] a derivation of the probability density $W\left(\widetilde{Q}_{0}, \widetilde{Q}_{1}, \widetilde{Q}_{2}\right)$ for the outcome of the triple measurement procedure, Appendix A 2; and (iii) a use of this probability density to show that the combination $\widetilde{R} \equiv \widetilde{Q}_{0}-2 \widetilde{Q}_{1}+\widetilde{Q}_{2}$ of the measurement results contains the desired information about $\bar{F}$ uncontaminated by any noise from the test-mass initial state, Appendix A 3.

\section{Single position measurement}

Let $|\Psi\rangle$ be the state of the test mass before the measurement and

$$
|\psi\rangle=\int_{-\infty}^{\infty} \psi(Q)|Q\rangle d Q
$$

be the initial state of the meter, where the meter's eigenstates are normalized by

$$
\left\langle Q^{\prime} \mid Q\right\rangle=\delta\left(Q-Q^{\prime}\right)
$$

We leave the test-mass state $|\Psi\rangle$ completely unspecified since our goal is to show that it has no influence at all on the measurement outcome. For concreteness we specify the meter's initial wave function $\psi(Q)$ to be Gaussian:

$$
\psi(Q)=\frac{1}{\sqrt{\sqrt{2 \pi} \Delta_{Q}}} \exp \left[-\frac{Q^{2}}{2 \Delta_{Q}^{2}}\left(\frac{1}{2}-\frac{i \Delta_{Q P}}{\hbar}\right)\right] .
$$

Here $\Delta_{Q}$ (denoted $\Delta Q^{\text {before }}$ in the text) is the initial variance of $Q$ and

$$
\Delta_{Q P}=\frac{\langle\hat{Q} \hat{P}+\hat{P} \hat{Q}\rangle}{2}
$$

is the initial cross correlation of the meter's position and momentum. For this Gaussian initial state, the variance $\Delta_{P}$ of the meter's momentum (denoted $\Delta P^{\text {before }}$ in the text) is given by the minimum-uncertainty relation 


$$
\Delta_{Q}^{2} \Delta_{P}^{2}-\Delta_{P Q}^{2}=\frac{\hbar^{2}}{4}
$$

The first stage of the measurement process is the interaction of the test mass and the meter. In the Schrödinger picture this interaction puts the meter and test mass into the entangled state

$$
\hat{U}|\psi\rangle|\Psi\rangle
$$

where

$$
\hat{U}=\exp \left(\frac{i \hat{x} \hat{P}}{\hbar}\right)
$$

is the evolution operator associated with the interaction (delta function) part of the Hamiltonian (2.14).

The next stage is a precise measurement of the meter's generalized position $\hat{Q}$. This measurement disentangles the quantum states of the test mass and meter: the meter gets reduced to the eigenstate $|\widetilde{Q}\rangle$ of $\hat{Q}$, where $\widetilde{Q}$ is the $c$ number obtained as a result of this measurement, and the test mass gets reduced to the state

$$
\frac{\langle\widetilde{Q}|\hat{U}| \psi\rangle|\Psi\rangle}{\sqrt{W(\widetilde{Q})}}=\frac{\hat{\Omega}(\widetilde{Q})|\Psi\rangle}{\sqrt{W(\widetilde{Q})}},
$$

where

$$
\hat{\Omega}(\widetilde{Q})=\langle\widetilde{Q}|\hat{U}| \psi\rangle
$$

is the reduction operator describing the entire two-stage measurement procedure, and

$$
W(\widetilde{Q})=\left\langle\Psi\left|\hat{\Omega}^{\dagger}(\widetilde{Q}) \hat{\Omega}(\widetilde{Q})\right| \Psi\right\rangle
$$

is the probability density for obtaining the result $\widetilde{Q}$.

An explicit form for the reduction operator can be obtained by substituting Eqs. (A1), (A3) and (A7) into Eq. (A9); the result is

$$
\begin{aligned}
\Omega(\widetilde{Q}) & =\left\langle\widetilde{Q}\left|\exp \left(\frac{i \hat{x} \hat{P}}{\hbar}\right) \int_{-\infty}^{\infty} \psi(Q)\right| Q\right\rangle d Q \\
& =\left\langle\widetilde{Q}\left|\int_{-\infty}^{\infty}\right| x\right\rangle\langle x|\psi(Q)| Q-x\rangle d x d Q \\
& =\int_{-\infty}^{\infty}|x\rangle\langle x| \psi(\widetilde{Q}+x) d x \\
& =\frac{1}{\sqrt{\sqrt{2 \pi} \Delta_{Q}}} \exp \left[-\frac{(\widetilde{Q}+\hat{x})^{2}}{2 \Delta_{Q}^{2}}\left(\frac{1}{2}-\frac{i \Delta_{Q P}}{\hbar}\right)\right],
\end{aligned}
$$

where we have used the shift-operator relation $e^{i \hat{x} \hat{P} / \hbar}|Q\rangle$ $=|Q-\hat{x}\rangle=\int_{-\infty}^{\infty} d x|x\rangle\langle x \mid Q-x\rangle$ and the relation $\langle\widetilde{Q} \mid Q-x\rangle$ $=\delta(Q-x-\widetilde{Q})$.

We will need below the following formulas (some are evident, and for the others we provide outlines of the proofs):

$\int_{-\infty}^{\infty} \hat{\Omega}^{\dagger}(\widetilde{Q}) \hat{\Omega}(\widetilde{Q}) d \widetilde{Q}=1$,

$\int_{-\infty}^{\infty} \hat{\Omega}^{\dagger}(\widetilde{Q}) \hat{\Omega}(\widetilde{Q}) \widetilde{Q} d \widetilde{Q}=-\hat{x}$

$\int_{-\infty}^{\infty} \hat{\Omega}^{\dagger}(\widetilde{Q}) \hat{\Omega}(\widetilde{Q}) \widetilde{Q}^{2} d \widetilde{Q}=\hat{x}^{2}+\Delta_{Q}^{2}$,

$\int_{-\infty}^{\infty} \hat{\Omega}^{\dagger}(\widetilde{Q}) \hat{x}^{n} \hat{\Omega}(\widetilde{Q}) d \widetilde{Q}=\hat{x}^{n} \quad(n=0,1, \ldots)$,

$\int_{-\infty}^{\infty} \hat{\Omega}^{\dagger}(\widetilde{Q}) \hat{x} \hat{\Omega}(\widetilde{Q}) \widetilde{Q} d \widetilde{Q}=-\hat{x}^{2}$,

$$
\begin{aligned}
\int_{-\infty}^{\infty} & \hat{\Omega}^{\dagger}(\widetilde{Q}) \hat{p} \hat{\Omega}(\widetilde{Q}) d \widetilde{Q} \\
& =\int_{-\infty}^{\infty} \hat{\Omega}^{\dagger}(\widetilde{Q})(\hat{\Omega}(\widetilde{Q}) \hat{p}+[\hat{p}, \hat{\Omega}(\widetilde{Q})]) d \widetilde{Q} \\
& =\int_{-\infty}^{\infty} \hat{\Omega}^{\dagger}(\widetilde{Q}) \hat{\Omega}(\widetilde{Q}) d \widetilde{Q} \hat{p}-i \hbar \int_{-\infty}^{\infty} \hat{\Omega}(\widetilde{Q}) \frac{d \hat{\Omega}^{\dagger}(\widetilde{Q})}{d \hat{x}} d \widetilde{Q} \\
& =\hat{p},
\end{aligned}
$$

$\int_{-\infty}^{\infty} \hat{\Omega}^{\dagger}(\widetilde{Q}) \hat{p}^{2} \hat{\Omega}(\widetilde{Q}) d \widetilde{Q}$

$=\int_{-\infty}^{\infty}\left(\hat{p} \hat{\Omega}^{\dagger}(\widetilde{Q})+\left[\hat{\Omega}^{\dagger}(\widetilde{Q}), \hat{p}\right]\right)(\hat{\Omega}(\widetilde{Q}) \hat{p}+[\hat{p}, \hat{\Omega}(\widetilde{Q})]) d \widetilde{Q}$

$=\hat{p} \int_{-\infty}^{\infty} \hat{\Omega}^{\dagger}(\widetilde{Q}) \hat{\Omega}(\widetilde{Q}) d \widetilde{Q} \hat{p}+\hbar^{2} \int_{-\infty}^{\infty} \frac{d \hat{\Omega}^{\dagger}(\widetilde{Q})}{d \hat{x}} \frac{d \hat{\Omega}(\widetilde{Q})}{d \hat{x}} d \widetilde{Q}$

$=\hat{p}^{2}+\frac{1}{\Delta_{Q}^{2}}\left(\frac{\hbar^{2}}{4}+\Delta_{Q P}^{2}\right)=\hat{p}^{2}+\Delta_{P}^{2}$,

$\int_{-\infty}^{\infty} \hat{\Omega}^{\dagger}(\widetilde{Q}) \hat{p} \hat{\Omega}(\widetilde{Q}) \widetilde{Q} d \widetilde{Q}$

$$
=\int_{-\infty}^{\infty} \hat{\Omega}^{\dagger}(\widetilde{Q})(\hat{\Omega}(\widetilde{Q}) \hat{p}+[\hat{p}, \hat{\Omega}(\widetilde{Q})]) \widetilde{Q} d \widetilde{Q}
$$




$$
\begin{aligned}
& =\int_{-\infty}^{\infty} \hat{\Omega}^{\dagger}(\widetilde{Q}) \hat{\Omega}(\widetilde{Q}) \widetilde{Q} d \widetilde{Q} \hat{p}-i \hbar \\
& \quad \times \int_{-\infty}^{\infty} \hat{\Omega}(\widetilde{Q}) \frac{d \hat{\Omega}^{\dagger}(\widetilde{Q})}{d \hat{x}} \widetilde{Q} d \widetilde{Q} \\
& =-\hat{x} \hat{p}+i \hbar\left(\frac{1}{2}-\frac{\Delta_{Q P}}{\hbar}\right)=-\frac{\hat{x} \hat{p}+\hat{p} \hat{x}}{2}+\Delta_{Q P}, \\
& \int_{-\infty}^{\infty} \hat{\Omega}^{\dagger}(\widetilde{Q})(\hat{x} \hat{p}+\hat{p} \hat{x}) \hat{\Omega}(\widetilde{Q}) d \widetilde{Q}=\hat{x} \hat{p}+\hat{p} \hat{x} .
\end{aligned}
$$

\section{The triple measurement procedure}

The triple measurement procedure described in Sec. II B 1 of the text consists of the following five stages.

(1) An initial position measurement of the type we have just analyzed, using meter number 0 . This measurement reduces the test mass's wave function to

$$
\frac{\widehat{\Omega}_{0}\left(\widetilde{Q}_{0}\right)|\Psi\rangle}{\sqrt{W_{0}\left(\widetilde{Q}_{0}\right)}}
$$

[Eq. (A8)], where $\hat{\Omega}_{0}\left(\widetilde{Q}_{0}\right)$ is the reduction operator [Eq. (A9)], and $\widetilde{Q}_{0}$ is the result of this measurement. The probability density for obtaining this result is equal to

$$
W_{0}\left(\widetilde{Q}_{0}\right)=\left\langle\Psi\left|\hat{\Omega}_{0}^{\dagger}\left(\widetilde{Q}_{0}\right) \hat{\Omega}_{0}\left(\widetilde{Q}_{0}\right)\right| \Psi\right\rangle
$$

[Eq. (A10)].

(2) Free evolution of the test mass during the time $\tau$. Denoting the corresponding evolution operator by $\hat{\mathcal{U}}_{0}$, the test-mass wave function after this stage is given by

$$
\frac{\hat{\mathcal{U}}_{0} \hat{\Omega}_{0}\left(\widetilde{Q}_{0}\right)|\Psi\rangle}{\sqrt{W_{0}\left(\widetilde{Q}_{0}\right)}} .
$$

(3) Second position measurement of the same type as in the first stage, but using a new meter, number 1 . The measurement result is denoted $\widetilde{Q}_{1}$, the reduction operator is $\Omega_{1}\left(\widetilde{Q}_{1}\right)$, and the measurement reduces the test-mass state to

$$
\frac{\hat{\Omega}_{1}\left(\widetilde{Q}_{1}\right) \hat{\mathcal{U}}_{0} \hat{\Omega}_{0}\left(\widetilde{Q}_{0}\right)|\Psi\rangle}{\sqrt{W_{1}\left(\widetilde{Q}_{0}, \widetilde{Q}_{1}\right)}},
$$

where

$$
W_{1}\left(\widetilde{Q}_{0}, \widetilde{Q}_{1}\right)=\left\langle\Psi\left|\hat{\Omega}_{0}^{\dagger}\left(\widetilde{Q}_{0}\right) \hat{\mathcal{U}}_{0}^{\dagger} \hat{\Omega}_{1}^{\dagger}\left(\widetilde{Q}_{1}\right) \hat{\Omega}_{1}\left(\widetilde{Q}_{1}\right) \hat{\mathcal{U}}_{0} \hat{\Omega}_{0}\left(\widetilde{Q}_{0}\right)\right| \Psi\right\rangle
$$

is the joint probability distribution for the first two measurement results, $\widetilde{Q}_{0}$ and $\widetilde{Q}_{1}$.
(4) Second free evolution of the test mass with the evolution operator $\hat{\mathcal{U}}_{1}$. After this stage the test-mass wave function is

$$
\frac{\hat{\mathcal{U}}_{1} \hat{\Omega}_{1}\left(\widetilde{Q}_{1}\right) \hat{\mathcal{U}}_{0} \hat{\Omega}_{0}\left(\widetilde{Q}_{0}\right)|\Psi\rangle}{\sqrt{W_{1}\left(\widetilde{Q}_{0}, \widetilde{Q}_{1}\right)}} .
$$

(5) Finally, a third position measurement using a new meter, number 2 , with the result $\widetilde{Q}_{2}$. After this measurement the test-mass state is

$$
\frac{\hat{\Omega}_{2}\left(\widetilde{Q}_{2}\right) \hat{\mathcal{U}}_{1} \hat{\Omega}_{1}\left(\widetilde{Q}_{1}\right) \hat{\mathcal{U}}_{0} \hat{\Omega}_{0}\left(\widetilde{Q}_{0}\right)|\Psi\rangle}{\sqrt{W_{2}\left(\widetilde{Q}_{0}, \widetilde{Q}_{1}, \widetilde{Q}_{2}\right)}},
$$

where

$$
\begin{aligned}
W_{2}\left(\widetilde{Q}_{0}, \widetilde{Q}_{1}, \widetilde{Q}_{2}\right)= & \langle\Psi| \hat{\Omega}_{0}^{\dagger}\left(\widetilde{Q}_{0}\right) \hat{\mathcal{U}}_{0}^{\dagger} \hat{\Omega}_{1}^{\dagger}\left(\widetilde{Q}_{1}\right) \hat{\mathcal{U}}_{1}^{\dagger} \hat{\Omega}_{2}^{\dagger}\left(\widetilde{Q}_{2}\right) \\
& \times \hat{\Omega}_{2}\left(\widetilde{Q}_{2}\right) \hat{\mathcal{U}}_{1} \hat{\Omega}_{1}\left(\widetilde{Q}_{1}\right) \hat{\mathcal{U}}_{0} \hat{\Omega}_{0}\left(\widetilde{Q}_{0}\right)|\Psi\rangle
\end{aligned}
$$

is the joint probability distribution for all three measurement outcomes.

Equation (A28) is the principal result of this subsection. We shall use it to study the statistics of the measurement outcomes. In that study we shall need the following expression for each of the three reduction operators [Eq. (A11)]:

$$
\hat{\Omega}_{s}\left(\widetilde{Q}_{s}\right)=\frac{1}{\sqrt{\sqrt{2 \pi} \Delta_{Q s}}} \exp \left[-\frac{\left(\widetilde{Q}_{s}+\hat{x}\right)^{2}}{2 \Delta_{Q s}^{2}}\left(\frac{1}{2}-\frac{i \Delta_{Q P s}}{\hbar}\right)\right],
$$

where $s=1,2,3$.

\section{Statistics of the measurement results}

If an explicit form for the initial wave function $|\Psi\rangle$ were specified, then the probability density (A28) could be calculated directly. However, that calculation would be very cumbersome, the final result would be quite complicated, and we have no need for it. Our final goal is not to study $W_{2}$, but rather to analyze the statistics of the quantity $\widetilde{R}=\widetilde{Q}_{0}-2 \widetilde{Q}_{1}$ $+\widetilde{Q}_{2}$, which the experimenter computes from the three measurement outcomes $\widetilde{Q}_{s}$ after the triple measurement procedure is complete. Specifically, we wish to verify the results of the text's Heisenberg-picture analysis: (i) That the mean value of $\widetilde{R}$ over a large number of experiments is $\langle\widetilde{R}\rangle$ $=\left(-\tau^{2} / \mu\right) \bar{F}$, where $\tau$ is the time between each pair of measurements, $\mu$ is the mass of the test mass, and $\bar{F}$ is the mean force that acts on the test mass [Eqs. (2.24) and (2.23) of the text]. (ii) That the variance of $\widetilde{R}$ (and thence of the measured value of $\bar{F})$ is independent of the test-mass initial state $|\Psi\rangle$, and is given by Eq. (2.25) when the meters' individual initial 
states have no position-momentum correlations, $\Delta_{Q P S}=0$, and can be made to vanish by a clever, "squeezed" choice of the meters' initial states.

a. Mean value. The mean value of $\widetilde{R}$ over a large number of experiments is determined by the joint probability distribution $W_{3}$ for the measurement outcomes:

$$
\begin{aligned}
\langle\widetilde{R}\rangle= & \left\langle\widetilde{Q}_{0}-2 \widetilde{Q}_{1}+\widetilde{Q}_{2}\right\rangle \\
= & \int_{-\infty}^{\infty}\left(\widetilde{Q}_{0}-2 \widetilde{Q}_{1}+\widetilde{Q}_{2}\right) \\
& \times W_{2}\left(\widetilde{Q}_{0}, \widetilde{Q}_{1}, \widetilde{Q}_{2}\right) d \widetilde{Q}_{0} d \widetilde{Q}_{1} d \widetilde{Q}_{2} .
\end{aligned}
$$

Using Eqs. (A12), (A13), we bring this into the form

$$
\begin{aligned}
\langle\widetilde{R}\rangle= & \int_{-\infty}^{\infty}\langle\Psi| \hat{\Omega}_{0}^{\dagger}\left(\widetilde{Q}_{0}\right) \hat{\mathcal{U}}_{0}^{\dagger} \hat{\Omega}_{1}^{\dagger}\left(\widetilde{Q}_{1}\right) \hat{\mathcal{U}}_{1}^{\dagger}\left(\widetilde{Q}_{0}-2 \widetilde{Q}_{1}-\hat{x}\right) \\
& \times \hat{\mathcal{U}}_{1} \hat{\Omega}_{1}\left(\widetilde{Q}_{1}\right) \hat{\mathcal{U}}_{0} \hat{\Omega}_{0}\left(\widetilde{Q}_{0}\right)|\Psi\rangle d \widetilde{Q}_{0} d \widetilde{Q}_{1} .
\end{aligned}
$$

Taking into account that

$$
\begin{gathered}
\mathcal{U}_{1}^{\dagger} \mathcal{U}_{1}=1, \\
\mathcal{U}_{1}^{\dagger} \hat{x} \mathcal{U}_{1}=x+\frac{\hat{p} \tau}{\mu}+x_{F \quad 1},
\end{gathered}
$$

where $\mu$ is the mass of the test mass and

$$
x_{F \quad 1}=\frac{1}{\mu} \int_{\tau}^{2 \tau}(2 \tau-t) F(t) d t
$$

is the displacement of the test mass during stage (4) (the second interval of free evolution) caused by the external force $F(t)$, expression (A31) can be further reduced to the form

$$
\begin{aligned}
\langle\widetilde{R}\rangle= & \int_{-\infty}^{\infty}\langle\Psi| \hat{\Omega}_{0}^{\dagger}\left(\widetilde{Q}_{0}\right) \mathcal{U}_{0}^{\dagger} \hat{\Omega}_{1}^{\dagger}\left(\widetilde{Q}_{1}\right)\left(\widetilde{Q}_{0}-2 \widetilde{Q}_{1}\right. \\
& \left.-\hat{x}-\frac{\hat{p} \tau}{\mu}-x_{F 1}\right) \hat{\Omega}_{1}\left(\widetilde{Q}_{1}\right) \hat{\mathcal{U}}_{0} \hat{\Omega}_{0}\left(\widetilde{Q}_{0}\right)|\Psi\rangle d \widetilde{Q}_{0} d \widetilde{Q}_{1} .
\end{aligned}
$$

The next calculations are just a repetition of the previous ones, with only the addition of Eqs. (A15), (A17) and

$$
\begin{gathered}
\mathcal{U}_{0}^{\dagger} \hat{x} \mathcal{U}_{0}=x+\frac{\hat{p} \tau}{\mu}+x_{F 0}, \\
\mathcal{U}_{0}^{\dagger} \hat{p} \mathcal{U}_{0}=p+p_{F 0},
\end{gathered}
$$

where

$$
\begin{gathered}
x_{F 0}=\frac{1}{\mu} \int_{0}^{\tau}(\tau-t) F(t) d t, \\
p_{F 0}=\int_{0}^{\tau} F(t) d t .
\end{gathered}
$$

They give

$$
\begin{aligned}
\langle\widetilde{R}\rangle & =\int_{-\infty}^{\infty}\left\langle\Psi\left|\hat{\Omega}_{0}^{\dagger}\left(\widetilde{Q}_{0}\right) \mathcal{U}_{0}^{\dagger}\left(\widetilde{Q}_{0}+2 \hat{x}-\hat{x}-\frac{\hat{p} \tau}{\mu}-x_{F 1}\right) \mathcal{U}_{0} \hat{\Omega}_{0}\left(\widetilde{Q}_{0}\right)\right| \Psi\right\rangle d \widetilde{Q}_{0} \\
& =\int_{-\infty}^{\infty}\left\langle\Psi\left|\hat{\Omega}_{0}^{\dagger}\left(\widetilde{Q}_{0}\right) \mathcal{U}_{0}^{\dagger}\left(\widetilde{Q}_{0}+\hat{x}+x_{F 0}-\frac{p_{F 0} \tau}{\mu}-x_{F 1}\right) \hat{\Omega}_{0}\left(\widetilde{Q}_{0}\right)\right| \Psi\right\rangle d \widetilde{Q}_{0} \\
& =\left\langle\Psi\left|\left(x_{F 0}-\frac{p_{F 0} \tau}{\mu}-x_{F 1}\right)\right| \Psi\right\rangle=x_{F 0}-\frac{p_{F 0} \tau}{\mu}-x_{F 1} \\
& =-\frac{1}{\mu} \int_{0}^{2 \tau}(\tau-|t-\tau|) F(t) d t \equiv-\frac{\tau^{2}}{\mu} \bar{F} .
\end{aligned}
$$

This agrees with the Heisenberg-picture prediction [Eqs. (2.24) and (2.23) of the text, where we must note that the meters' initial states have $\left.\left\langle Q_{s}\right\rangle=\left\langle P_{s}\right\rangle=0\right]$.

$b$. Variance. The mean square value of the measurement outcome $\widetilde{R}$ over a large number of experiments is given by 


$$
\left\langle\widetilde{R}^{2}\right\rangle=\left\langle\left(\widetilde{Q}_{0}-2 \widetilde{Q}_{1}+\widetilde{Q}_{2}\right)^{2}\right\rangle=\int_{-\infty}^{\infty}\left(\widetilde{Q}_{0}-2 \widetilde{Q}_{1}+\widetilde{Q}_{2}\right)^{2} W_{2}\left(\widetilde{Q}_{0}, \widetilde{Q}_{1}, \widetilde{Q}_{2}\right) d \widetilde{Q}_{0} d \widetilde{Q}_{1} d \widetilde{Q}_{2}
$$

Using Eqs. (A12)-(A20), (A32), (A33), (A36), and (A37), we obtain

$$
\begin{aligned}
\left\langle\widetilde{R}^{2}\right\rangle & =\left\langle\left(\widetilde{Q}_{0}-2 \widetilde{Q}_{1}+\widetilde{Q}_{2}\right)^{2}\right\rangle \\
& =\int_{-\infty}^{\infty}\left\langle\Psi\left|\hat{\Omega}_{0}^{\dagger}\left(\widetilde{Q}_{0}\right) \hat{\mathcal{U}}_{0}^{\dagger} \hat{\Omega}_{1}^{\dagger}\left(\widetilde{Q}_{1}\right) \hat{\mathcal{U}}_{1}^{\dagger}\left[\left(\widetilde{Q}_{0}-2 \widetilde{Q}_{1}-\hat{x}\right)^{2}+\Delta_{Q}^{2}\right] \hat{\mathcal{U}}_{1} \hat{\Omega}_{1}\left(\widetilde{Q}_{1}\right) \hat{\mathcal{U}}_{0} \hat{\Omega}_{0}\left(\widetilde{Q}_{0}\right)\right| \Psi\right\rangle d \widetilde{Q}_{0} d \widetilde{Q}_{1} \\
& =\int_{-\infty}^{\infty}\left\langle\Psi\left|\hat{\Omega}_{0}^{\dagger}\left(\widetilde{Q}_{0}\right) \mathcal{U}_{0}^{\dagger} \hat{\Omega}_{1}^{\dagger}\left(\widetilde{Q}_{1}\right)\left[\left(\widetilde{Q}_{0}-2 \widetilde{Q}_{1}-\hat{x}-\frac{\hat{p} \tau}{m}-x_{F 1}\right)^{2}+\Delta_{Q 2}^{2}\right] \hat{\Omega}_{1}\left(\widetilde{Q}_{1}\right) \hat{\mathcal{U}}_{0} \hat{\Omega}_{0}\left(\widetilde{Q}_{0}\right)\right| \Psi\right\rangle d \widetilde{Q}_{0} d \widetilde{Q}_{1} \\
& =\int_{-\infty}^{\infty}\left\langle\Psi\left|\hat{\Omega}_{0}^{\dagger}\left(\widetilde{Q}_{0}\right) \mathcal{U}_{0}^{\dagger}\left[\left(\widetilde{Q}_{0}+\hat{x}-\frac{\hat{p} \tau}{m}-x_{F 1}\right)^{2}+4 \Delta_{Q 1}^{2}+\frac{4 \Delta_{Q P 1} \tau}{m}+\left(\frac{\Delta_{P 1} \tau}{\mu}\right)^{2}+\Delta_{Q 2}^{2}\right] \hat{\mathcal{U}}_{0} \hat{\Omega}_{0}\left(\widetilde{Q}_{0}\right)\right| \Psi\right\rangle d \widetilde{Q}_{0} \\
& =\int_{-\infty}^{\infty}\left\langle\Psi\left|\hat{\Omega}_{0}^{\dagger}\left(\widetilde{Q}_{0}\right)\left[\left(\widetilde{Q}_{0}+\hat{x}+x_{F 0}-\frac{p_{F 0} \tau}{m}-x_{F 1}\right)^{2}+4 \Delta_{Q 1}^{2}+\frac{4 \Delta_{Q P 1} \tau}{m}+\left(\frac{\Delta_{P 1} \tau}{\mu}\right)^{2}+\Delta_{Q 2}^{2}\right] \hat{\Omega}_{0}\left(\widetilde{Q}_{0}\right)\right| \Psi\right\rangle d \widetilde{Q}_{0} \\
& =\left\langle\Psi\left|\left[\left(x_{F 0}-\frac{p_{F 0} \tau}{m}-x_{F 1}\right)^{2}+\Delta_{Q 0}^{2}+4 \Delta_{Q 1}^{2}+\frac{4 \Delta_{Q P 1} \tau}{m}+\left(\frac{\Delta_{P 1} \tau}{\mu}\right)^{2}+\Delta_{Q 2}^{2}\right]\right| \Psi\right\rangle \\
& =\left\langle\widetilde{Q}_{0}-2 \widetilde{Q}_{1}+\widetilde{Q}_{2}\right\rangle^{2}+\Delta_{Q 0}^{2}+4 \Delta_{Q 1}^{2}+\frac{4 \Delta_{Q P 1} \tau}{m}+\left(\frac{\Delta_{P 1} \tau}{\mu}\right)^{2}+\Delta_{Q 2}^{2} .
\end{aligned}
$$

Subtracting off the square of the mean, $\langle\widetilde{R}\rangle^{2}=\left\langle\widetilde{Q}_{0}-2 \widetilde{Q}_{1}\right.$ $\left.+\widetilde{Q}_{2}\right\rangle^{2}$, we obtain for the variance of the computed quantity $\widetilde{R}$, over many experiments,

$$
\begin{aligned}
\frac{\tau^{4}}{\mu^{2}}(\Delta \bar{F})^{2} & =(\Delta \widetilde{R})^{2}=\left\langle\hat{R}^{2}\right\rangle-\langle R\rangle^{2} \\
& =\Delta_{Q 0}^{2}+4 \Delta_{Q 1}^{2}+\frac{4 \Delta_{Q P 1} \tau}{m}+\left(\frac{\Delta_{P 1} \tau}{\mu}\right)^{2}+\Delta_{Q 2}^{2} ;
\end{aligned}
$$

see Eq. (A40) for the first equality. This variance is independent of the test-mass initial state $|\Psi\rangle$, in accord with prediction of the Heisenberg-picture analysis [passage following Eq. (2.22) of the text]. When the three meters are all prepared in "naive" initial states, i.e. in states with uncorrelated generalized position $\hat{Q}_{s}$ and momentum $\hat{P}_{s}$, i.e. when $\Delta_{Q P s}=0$, then the variance (A43) has the form that we deduced using the Heisenberg picture [Eq. (2.25)]. When the meters are prepared in the more clever "squeezed" manner, i.e. in near eigenstates of $\hat{Q}_{0}, \hat{Q}_{1}^{\text {squeeze }}=\hat{Q}_{1}-\hat{P}_{1} \tau / 2 \mu$ and $\hat{Q}_{2}$, then the variance (A43) vanishes, in accord with the Heisenberg-picture prediction [passage following Eq. (2.28)].

\section{APPENDIX B: LINEAR MEASUREMENTS}

An important feature of our pedagogical examples (Sec. II), and of measurements performed by interferometric gravitational-wave detectors, is that they all are linear measurements in the sense of Ref. [3]; i.e., they all satisfy the following two conditions:

(i) Linearity of the output. The meter's output can be written as the sum of the operator for the test object's measured variable and the operator for the meter's additive noise [cf. Eq. (2.4a)], and the additive noise does not depend on the initial state of the test object. Formally this sum is an operator, but it can be treated as a classical variable because it turns out to commute with itself at different times.

(ii) Linearity of the back action. The measurementinduced perturbations of all the test-object observables that are involved in the measurement procedure can be described by linear formulas similar to Eq. (2.4b), and the perturbations [e.g. the second term on the right side of (2.4b)] do not depend on the initial state of the test object.

This second condition requires discussion: The perturbations' independence of the test-object initial state is particularly important when several test-object variables are measured consecutively-for example, if the same HeisenbergPicture variable is measured quickly and repetitively at different moments of time as in our pedagogical examples (Sec. II), or if a variable is measured continuously as in a gravitational-wave detector (Sec. III). Suppose, for example, that the variable $\hat{x}_{1}$ is measured with precision $\Delta x_{1}^{\text {meas }}$ thereby perturbing, via back-action, some other variable $\hat{x}_{2}$. Then the accuracy of a subsequent measurement of $\hat{x}_{2}$ will be constrained by the perturbation 


$$
\Delta x_{2}^{\text {pert }}=\frac{\hbar}{2 \Delta x_{1}^{\text {meas }}}\left|\left\langle\left[\hat{x}_{1}, \hat{x}_{2}\right]\right\rangle\right| .
$$

Our condition (ii) of back-action linearity requires that this perturbation not depend on the initial state of the test object. A sufficient condition for this is that the commutator $\left[\hat{x}_{1}, \hat{x}_{2}\right]$ be a $c$-number, and that this requirement be fulfilled for all the operators involved in the measurement. ${ }^{6}$

Linear measurements are closely related to linear systems (those for which the equations of motion for the generalized coordinates and momenta are linear; for example, a free mass and a harmonic oscillator) because the commutators of such systems' coordinates and momenta are $c$-numbers.

In nonlinear measurements (e.g. measurements of a particle in a double-welled potential), some very strange phenomena can arise, for example the quantum Zeno effect.

Strictly speaking, all real meters are nonlinear. However, in most cases they can be regarded as linear to high accuracy. For example, if one measures displacements of a mirror of a Fabry-Perot cavity by monitoring the phase of light that passes through the cavity (as is done in LIGO), then the measurements are linear so long as the displacements are much smaller than the width of a cavity resonance, i.e. much smaller than $\lambda / \mathcal{F}$ where $\lambda$ is the wavelength of the light and $\mathcal{F}$ is the cavity finesse.

If, by contrast, the displacements are comparable to or much larger than $\lambda / \mathcal{F}$, then the measurements are strongly nonlinear. An example is a proposed null-detector technique [37] for measuring the phase of a mechanical oscillator, in which the oscillating mass is an end mirror of a Fabry-Perot cavity, and the times at which the mirror passes through cavity-resonant positions are measured with high accuracy by the cavity's momentary transmissivity. These measurements are highly nonlinear because, in the proposed design, not only are the mirror displacements large compared to the cavity's linearity regime, $\lambda / \mathcal{F}$; the mechanical oscillator's amplitude of zero-point oscillations $\delta x_{\mathrm{zp}}$ is also large compared to $\lambda / \mathcal{F}$. State reduction plays an important role in this null detector's measurements: it drives the mechanical oscillator into a squeezed-phase state, thereby facilitating a highprecision monitoring of the oscillator's phase [37]. It would be instructive to analyze the use of this highly nonlinear meter to monitor a classical force that acts on the oscillator's mass. Does the oscillator's initial quantum state influence the accuracy of the monitoring?

Three properties of an interferometric gravitational-wave detector (interferometric position meter) allow one to consider it as linear with sufficiently high precision to justify the linear analysis given in this paper. First, its test-mass mirrors can be regarded as free masses (or as harmonic oscillators if significant electromagnetic rigidity exists in the system). Second, its linearity range $\lambda / \mathcal{F} \sim 10^{-6} \mathrm{~cm}$ is much greater than the wave-induced displacements of the test masses

\footnotetext{
${ }^{6}$ It can be shown that a slightly weaker condition is sufficient: second-order commutation of all these operators, $\left[\hat{x}_{i},\left[\hat{x}_{j}, \hat{x}_{k}\right]\right]=0$ for all $i, j, k$.
}

$\left(\lesssim 10^{-15} \mathrm{~cm}\right)$. Hence, the signal phase shift of the output optical beam depends linearly on the displacement. Third, the measurement of the photon flux out the dark port is virtually equivalent to the measurement of the phase of the output beam because (i) the signal phase shift is much less than one radian and (ii) the mean value of the amplitude of the optical pumping field is much larger than the quantum uncertainties of its quadrature amplitudes.

For a detailed presentation of the theory of linear measurements see Chaps. 5 and 6 of Ref. [3]. For a detailed application of this theory to interferometric gravitationalwave detectors see Ref. [17].

\section{APPENDIX C: VANISHING SELF-COMMUTATOR OF THE PHOTON NUMBER FLUX}

For any light beam (or other electromagnetic wave with confined cross section), the number flux operator at some chosen transverse plane (e.g. the entry to a photodetector) is

$$
\hat{\mathcal{N}}(t)=\int_{0}^{\infty} \frac{d \omega}{2 \pi} \int_{0}^{\infty} \frac{d \omega^{\prime}}{2 \pi} \hat{a}_{\omega}^{\dagger} \hat{a}_{\omega^{\prime}} e^{i\left(\omega-\omega^{\prime}\right) t} .
$$

Here $\hat{a}_{\omega}^{\dagger}$ is the creation operator and $\hat{a}_{\omega}$ the annihilation operator for photons of frequency $\omega$, and their commutators are

$$
\left[\hat{a}_{\omega}, \hat{a}_{\omega^{\prime}}\right]=\left[\hat{a}_{\omega}^{\dagger}, \hat{a}_{\omega^{\prime}}^{\dagger}\right]=0,\left[\hat{a}_{\omega}, \hat{a}_{\omega^{\prime}}^{\dagger}\right]=2 \pi \delta\left(\omega-\omega^{\prime}\right) .
$$

It is straightforward to verify from Eqs. (C1) and (C2) that

$$
\left[\hat{\mathcal{N}}(t), \hat{\mathcal{N}}\left(t^{\prime}\right)\right]=0
$$

Although this result is completely general, it is instructive to derive the vanishing self commutator for the specialized type of light beam that is used in interferometers and other force-measuring devices: a beam consisting of a monochromatic carrier with frequency $\omega_{o}$ plus sidebands embodied in $\hat{a}_{\omega}$ and $\hat{a}_{\omega}^{\dagger}$. In this case to high accuracy we can linearize in the product of the carrier field and the side-band fields, obtaining for the relevant (side-band) photon flux

$$
\hat{\mathcal{N}}_{1}(t)=\sqrt{\mathcal{N}}_{0}\left[\hat{a}(t)+\hat{a}^{\dagger}(t)\right]
$$

Here [in the notation of Eqs. (2.34) $-(2.37)] \mathcal{N}_{0}=A_{0}^{2}$ is the carrier's photon flux and $\hat{a}(t), \hat{a}^{\dagger}(t)$ are the time-domain side-band annihilation and creation operators with commutation relations [time-domain versions of Eq. (C2)]

$$
\begin{gathered}
{\left[\hat{a}(t), \hat{a}\left(t^{\prime}\right)\right]=0, \quad\left[\hat{a}^{\dagger}(t), \hat{a}^{\dagger}\left(t^{\prime}\right)\right]=0,} \\
{\left[\hat{a}(t), \hat{a}^{\dagger}\left(t^{\prime}\right)\right]=\delta\left(t-t^{\prime}\right) .}
\end{gathered}
$$

It is straightforward, using these commutation relations, to verify that

$$
\left[\mathcal{N}_{1}(t), \mathcal{N}_{1}\left(t^{\prime}\right)\right]=0
$$


It is interesting to note that, although the photon number flux self commutes, the energy flux (energy passing a fixed transverse surface per unit time)

$$
\hat{\mathcal{E}}(t)=\hbar \int_{0}^{\infty} d \omega \int_{0}^{\infty} d \omega^{\prime} \sqrt{\omega \omega^{\prime}} \hat{a}_{\omega^{\dagger}}^{\dagger} \hat{a}_{\omega^{\prime}} e^{i \omega\left(t-t^{\prime}\right)}
$$

does not self-commute,

$$
\left[\hat{\mathcal{E}}(t), \hat{\mathcal{E}}\left(t^{\prime}\right)\right] \neq 0
$$

This can be thought of as due to the energy-time uncertainty relation for photons. On the other hand, when (as in gravitational-wave interferometers) the light consists of a monochromatic carrier plus signals encoded in side bands with frequency $\Omega=\omega-\omega_{o} \ll \omega_{o}$, then for all practical purposes, $\hat{\mathcal{E}}(t)$ does self commute.
[1] V.B. Braginsky, Sov. Phys. JETP 26, 831 (1968); in Physical Experiments with Test Bodies, NASA Technical Translation TT F-672 (U.S. Technical Information Service, Springfied, VA, 1972).

[2] V.B. Braginsky and Yu.I. Vorontsov, Sov. Phys. Usp. 17, 644 (1975).

[3] V.B. Braginsky and F.Ya. Khalili, Quantum Measurement (Cambridge University Press, Cambridge, England, 1992).

[4] V.B. Braginsky, Yu.I. Vorontsov, and F.Ya. Khalili, Sov. Phys. JETP 46, 705 (1977).

[5] V.B. Braginsky and F.Ya. Khalili, Rev. Mod. Phys. 68, 1 (1996).

[6] S.P. Vyatchanin and E.A. Zubova, Phys. Lett. A 203, 269 (1995).

[7] S.P. Vyatchanin and A.B. Matsko, JETP 82, 1007 (1996).

[8] S.P. Vyatchanin and A.B. Matsko, JETP 83, 690 (1996).

[9] S.P. Vyatchanin and A.Yu. Lavrenov, Phys. Lett. A 231, 38 (1997).

[10] C.M. Caves, in Quantum Measurement and Chaos, edited by E.R. Pike (Plenum, New York, 1987).

[11] K.S. Thorne, in 300 Years of Gravitation, edited by S.W. Hawking and W. Israel (Cambridge University Press, Cambridge, England, 1987).

[12] A.F. Pace, M.J. Collett, and D.F. Walls, Phys. Rev. A 47, 3173 (1993).

[13] H.J. Kimble, Yu. Levin, A.B. Matsko, K.S. Thorne, and S.P. Vyatchanin, Phys. Rev. D 65, 022002 (2002).

[14] E. Gustafson, D. Shoemaker, K. Strain, and R. Weiss, LSC White Paper on Detector Research and Development, LIGO document T990080-00-D, 1999; available along with other relevant information at http://www.ligo.caltech.edu/ ligo2/

[15] A. Buonanno and Y. Chen, Class. Quantum Grav. 18, L1 (2001).

[16] A. Buonanno and Y. Chen, Phys. Rev. D 64, 042006 (2001).

[17] A. Buonanno and Y. Chen, Phys. Rev. D 65, 042001 (2002).

[18] W.G. Unruh, in Quantum Optics, Experimental Gravitation, and Measurement Theory, edited by P. Meystre and M.O. Scully (Plenum, New York, 1982), p. 647.

[19] V.B. Braginsky, M.L. Gorodetsky, and F.Ya. Khalili, Phys. Lett. A 232, 340 (1997).

[20] V.B. Braginsky, M.L. Gorodetsky, and F.Ya. Khalili, Phys. Lett. A 246, 485 (1998).
[21] V.B. Braginsky, M.L. Gorodetsky, F.Ya. Khalili, and K.S. Thorne, Phys. Rev. D 61, 044002 (2000).

[22] V.B. Braginsky and F.Ya. Khalili, Phys. Lett. A 257, 241 (1999).

[23] F.Ya. Khalili, Phys. Lett. A 288, 25 (2001).

[24] P. Purdue, Phys. Rev. D 66, 022001 (2002); P. Purdue and Y. Chen, ibid. 66, 122004 (2002).

[25] For this type of derivation applied to a harmonic oscillator see, e.g., K.S. Thorne, R.W.P. Drever, C.M. Caves, M. Zimmermann, and V.D. Sandberg, Phys. Rev. Lett. 40, 667 (1978); also J.N. Hollenhorst, Phys. Rev. D 19, 1669 (1979).

[26] C.M. Caves, Phys. Rev. D 33, 1643 (1986); 35, 1815 (1987).

[27] M.B. Mensky, Phys. Rev. D 20, 384 (1979); Sov. Phys. JETP 50, 667 (1979); Quantum Measurement and Decoherence (Kluwer Academic, Dordrecht, 2000).

[28] C.M. Caves, Phys. Rev. Lett. 45, 75 (1980).

[29] C.M. Caves, Phys. Rev. D 23, 1693 (1981).

[30] J. von Neumann, Mathematische Grundlagen der Quantenmechanik (Springer, Berlin, 1932), especially Chap. 6. [English translation: Mathematical Foundations of Quantum Mechanics (Princeton University Press, Princeton, NJ, 1955).]

[31] C.M. Caves and G.J. Milburn, Phys. Rev. A 36, 5543 (1987).

[32] See the Collett-Gardiner model problem presented in Sec. 3.2 of C.W. Gardiner, Quantum Noise (Springer-Verlag, Berlin, 1991). By combining Eqs. (3.2.27) and (3.2.28) of this reference, one deduces that $\left[\hat{A}_{\text {out }}(t), \hat{A}_{\text {out }}\left(t^{\prime}\right)\right]=0$ for all $t, t^{\prime}-\mathrm{a}$ result not explicitly discussed by Gardiner but closely related to issues that he does discuss. We thank Gardiner for calling our attention to this example.

[33] O. Alter and Y. Yamamoto, Phys. Lett. A 263, 226 (1999).

[34] O. Alter and Y. Yamamoto, Quantum Measurement of a Single System (Wiley, New York, 2001), Chap. 7.

[35] Equation (8.1.44) of Gardiner [32].

[36] V.B. Braginsky, V.P. Mitrofanov, and K.V. Tokmakov, Phys. Lett. A 218, 164 (1996); K.V. Tokmakov, V.P. Mitrofanov, V.B. Braginsky, S. Rowan, and J. Hough, in Gravitational Waves: Third Edoardo Amaldi Conference, edited by S. Meskkov, AIP Conf. Proc. No. 523 (AIP, Melville, NY, 2000).

[37] S.P. Vyatchanin, Vestn. Mosk. Univ., Ser. 3: Fiz., Astron. 20, 103 (1979); V.B. Braginsky, F.Ya. Khalili, and A.A. Kulaga, Phys. Lett. A 202, 1 (1995); A.A. Kulaga, ibid. 202, 7 (1995). 